\title{
The ROSAT-ESO Flux Limited X-ray (REFLEX) Galaxy cluster survey
}

\section{The cluster catalogue $e^{\star \star \star \star, \star \star \star}$}

\author{
H. Böhringer ${ }^{1}$, P. Schuecker ${ }^{1}$, L. Guzzo ${ }^{2}$, C. A. Collins ${ }^{3}$, W. Voges ${ }^{1}$, R. G. Cruddace ${ }^{4}$, \\ A. Ortiz-Gil ${ }^{5}$, G. Chincarini ${ }^{2,6}$, S. De Grandi ${ }^{2}$, A. C. Edge ${ }^{7}$, H. T. MacGillivray ${ }^{8}$, \\ D. M. Neumann ${ }^{9}$, S. Schindler ${ }^{10}$, and P. Shaver ${ }^{11}$
}

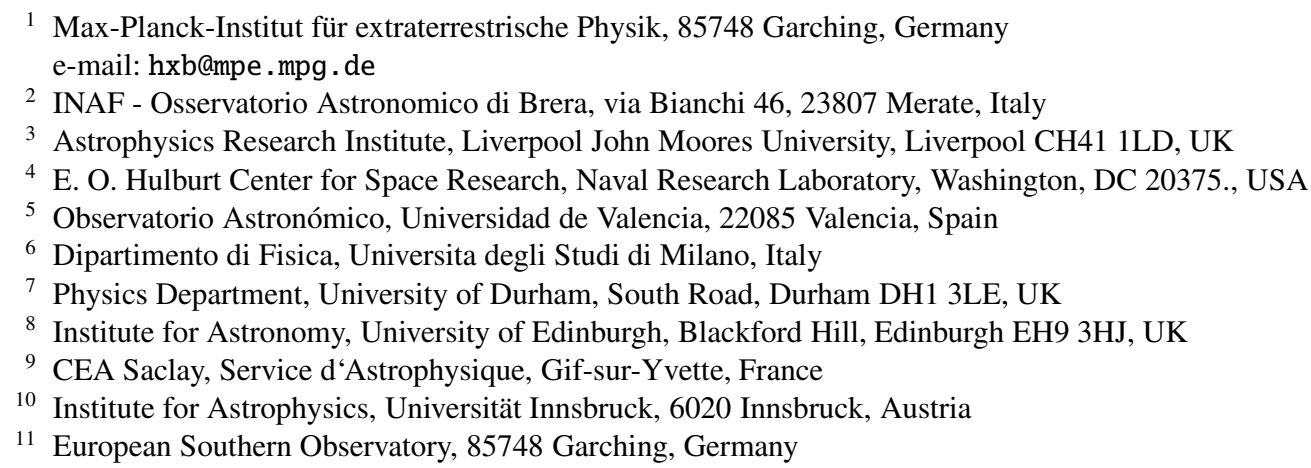

Received 9 October 2003 / Accepted 11 March 2004

\begin{abstract}
We present the catalogue of the REFLEX Cluster Survey providing information on the X-ray properties, redshifts, and some identification details of the clusters in the REFLEX sample. The catalogue describes a statistically complete X-ray flux-limited sample of 447 galaxy clusters above an X-ray flux of $3 \times 10^{-12} \mathrm{erg} \mathrm{s}^{-1} \mathrm{~cm}^{-2}$ (0.1 to $2.4 \mathrm{keV}$ ) in an area of 4.24 ster in the southern sky. The cluster candidates were first selected by their X-ray emission in the ROSAT-All Sky Survey and subsequently spectroscopically identified in the frame of an ESO key programme. Previously described tests have shown that the sample is more than $90 \%$ complete and there is a conservative upper limit of $9 \%$ on the fraction of clusters with a dominant X-ray contamination from AGN. In addition to the cluster catalogue we also describe the complete selection criteria as a function of the sky position and the conversion functions used to analyse the X-ray data. These are essential for the precise statistical analysis of the large-scale cluster distribution. This data set is at present the largest, statistically complete X-ray galaxy cluster sample. Together with these data set we also provide for the first time the full three-dimensional selection function. The sample forms the basis of several cosmological studies, one of the most important applications being the assessment of the statistics of the large-scale structure of the universe and the test of cosmological models. Part of these cosmological results have already been published.
\end{abstract}

Key words. catalogs - surveys - galaxies: clusters: general - cosmology: large-scale structure of Universe $\mathrm{X}$-rays: general - X-rays: galaxies: clusters

\footnotetext{
* Based on observations at the European Southern Observatory La Silla, Chile.

$\star \star$ The full Tables 2-9 are only available in electronic form at the CDS via anonymous ftp to

cdsarc.u-strasbg.fr $(130.79 .128 .5)$ or via

http://cdsweb.u-strasbg.fr/cgi-bin/qcat?J/A+A/425/367

as well as on our home page

http://www. xray.mpe.mpg.de/theorie/REFLEX/DATA

$\star \star \star$ Appendix is only available in electronic form at

http://www. edpsciences.org
}

\section{Introduction}

Clusters of galaxies are the largest building blocks of our Universe that can still reasonably well be characterized as unique objects. This makes them on one hand very important large-scale astrophysical laboratories in which a large variety of astrophysical processes can be studied in well characterized environments. For these laboratories we can measure for example their total gravitational mass, their matter composition, the internal gas density, temperature, and pressure of the 
intergalactic medium, their distance, and other important properties. The best basis for such astrophysical studies is a well documented catalogue of galaxy clusters to choose the best suited objects for the prospective study (e.g., Böhringer et al. 2001b).

On the other hand X-ray selected galaxy clusters are very good tracers of the large-scale structure of the Universe. Since there is a quite well understood relation between the distribution of galaxy clusters with known mass and the dark matter distribution, the statistics of the large-scale matter distribution in the Universe can be derived from the distribution of clusters in a well selected, statistically complete sample. This study of the large-scale structure was the main objective for the construction of the REFLEX sample. Several results on the construction of the sample (Böhringer et al. 2001a, Paper I), the assessment of the large-scale structure (Collins et al. 2000, Paper II; Schuecker et al. 2001a, Paper III; Böhringer et al. 2002, Paper IV; Schuecker et al. 2002, 2003a (Papers VI and VII), 2003b; Kerscher et al. 2001), on the statistics of substructure in REFLEX clusters Schuecker et al. (2001b), on the statistics of the cluster galaxy velocity dispersions (Ortiz-Gil et al. 2003), and on the X-ray temperatures of the most luminous, distant REFLEX clusters (Zhang et al. 2003) have already been published. Several further papers are in preparation.

Due to the close correlation of X-ray luminosity and mass for clusters of galaxies (e.g., Reiprich \& Böhringer 2002) the detection and selection of the sample clusters is currently best performed through the cluster X-ray emission. The ROSAT All-Sky Survey (RASS), which is still the only all-sky or wide-angle X-ray survey performed with an imaging X-ray telescope, is by far the best basis for such cosmological studies. It has been used previously in several projects to construct statistical galaxy cluster samples (Pierre et al. 1994; Romer et al. 1994; Ebeling et al. 1996, 1998, 2000; Burns et al. 1996; De Grandi et al. 1999; Ledlow et al. 1999; Böhringer et al. 2000, 2001c; Henry et al. 2001; Cruddace et al. 2002, 2003; Ebeling et al. 2001, 2002; Gioia et al. 2003). Part of these projects were studies connected to and profiting from the REFLEX survey program. None of the previous projects covers an area in the southern sky as large as REFLEX, except for the XBACS Abell cluster survey (Ebeling et al. 1996), which is shallower and restricted to those clusters previously identified by Abell (1958) and Abell et al. (1989).

The REFLEX catalogue of 447 clusters provides presently the largest statistically complete X-ray cluster sample. The volume of the Universe that is probed is larger than that covered by any present galaxy redshift survey except for the Sloan Digital Sky Survey, which goes to a slightly larger depth but will only cover about half the sky area of that covered by REFLEX, when completed.

The paper is organized as follows. In Sect. 2 we describe the survey and the selection characteristics. Section 3 provides a brief description of the X-ray data reduction and Sect. 4 describes the redshift determination and the cluster galaxy redshift statistics. The main catalogue is presented in Sect. 5 and some of its properties are reviewed in Sect. 6. In the latter section we also provide the numerical data and the recipe to construct the survey selection function in one and two dimensions
Table 1. Overview on the data presented in this paper in tabular form.

\begin{tabular}{|c|c|}
\hline Table & Content \\
\hline 2 & Count rate to flux conversion (for $z=0$ ). \\
\hline 3 & $\mathrm{~K}$-correction as a function of $z$ and $T_{\mathrm{x}}$ \\
\hline 4 & $\begin{array}{l}\text { Flux conversion from the } 0.1-2.4 \mathrm{keV} \text { to } \\
\text { the } 0.5 \text { to } 2.0 \mathrm{keV} \text { band. }\end{array}$ \\
\hline 5 & $\begin{array}{l}\text { Flux conversion from the } 0.1-2.4 \mathrm{keV} \text { band } \\
\text { to bolometric flux. }\end{array}$ \\
\hline 6 & REFLEX cluster catalogue for $h=0.7$ and $\Lambda$-cosmology. \\
\hline 7 & Further X-ray parameters of the REFLEX clusters. \\
\hline 8 & Sky coverage as a function of the flux limit. \\
\hline 9 & Angular modulation of the survey selection function. \\
\hline 10 & Close cluster pairs in the REFLEX sample. \\
\hline 11 & Clusters with multiple maxima in the REFLEX sample. \\
\hline 12 & $\begin{array}{l}\text { Line-of-sight redshift clustering at the } \\
\text { position of REFLEX clusters. }\end{array}$ \\
\hline
\end{tabular}

for any flux limit equal to or above the nominal REFLEX flux limit. Section 7 lists close cluster pairs and clusters with double or multiple X-ray maxima found in the REFLEX catalogue, and we describe in more detail those clusters where multiple redshift clustering is observed in the line-of-sight of the X-ray source. In Sect. 8 we compare the results with the previously derived survey samples and, finally, in Sect. 9 we provide a summary and conclusions. The Appendix provides further information on the identification and properties of some individual clusters. Table 1 gives an overview of the information presented in this paper in tabular form.

The luminosities and other cluster parameters which depend on the distance scale are derived for a Hubble constant of $H_{0}=70 \mathrm{~km} \mathrm{~s}^{-1} \mathrm{Mpc}^{-1}$ and a cosmological model with $\Omega_{\mathrm{m}}=0.3$ and $\Omega_{\Lambda}=0.7$ in the main tables of the paper. We also give in complementary tables provided only in electronic form the cluster properties for the previously most often used Einstein-de Sitter model with $H_{0}=50 \mathrm{~km} \mathrm{~s}^{-1} \mathrm{Mpc}^{-1}$, $\Omega_{\mathrm{m}}=1.0$ and $\Omega_{\Lambda}=0$ for an easier comparison with previous literature results.

\section{The REFLEX survey}

The construction of the REFLEX cluster sample is described in detail in Paper I. The survey area covers the southern sky up to declination $\delta=+2.5^{\circ}$, avoiding the band of the Milky Way $\left(\left|b_{I I}\right| \leq 20^{\circ}\right)$ and the regions of the Magellanic clouds. The total survey area is $13924 \mathrm{deg}^{2}$ or $4.24 \mathrm{sr}$. The regions that have been excised are defined in Table 2 in Paper I.

The X-ray detection of the clusters is based on the second processing of the RASS (RASS II, Voges et al. 1999), providing 54076 sources in the REFLEX area. Note that the public RASS catalogue available through the internet (http://www.xray.mpe.mpg.de/rosat/survey/ rass-bsc/; http://www . xray . mpe.mpg.de/rosat/survey/ rass-fsc/) is based on the third processing of the RASS which has a more complete sky coverage. Since we have frozen the cluster candidate list in 1998 near the end of the optical 
follow-up program, the cluster selection of the present cluster catalogue is based on RASS II. We have, however, checked that the X-ray properties of the individual catalogued objects are still consistent with the results obtained with the new processing. We have in particular redetermined all cluster centers by applying our analysis software to the RASS III data base (to ensure that the naming convention will be stable when finally basing all the analysis on RASS III). The positional changes were small in relation to the extent of the X-ray source. The second, enlarged version of the REFLEX sample with a lower flux limit will be based completely on RASS III.

All sources were reanalysed by means of the growth curve analysis (GCA) method (Böhringer et al. 2000) and the results are used to produce a flux-limited sample of RASS sources with a nominal flux of $F_{\mathrm{n}} \geq 3 \times 10^{-12} \mathrm{erg} \mathrm{s}^{-1} \mathrm{~cm}^{-2}$ (with $F_{\mathrm{n}}$ as defined below). This redetermination of the fluxes has been shown to be crucial for a precise measure of the fluxes of extended sources, as are the majority of the REFLEX clusters (Ebeling et al. 1996; De Grandi et al. 1997; Böhringer et al. 2000). Cluster candidates were found using a machine based correlation of these X-ray sources with galaxy density enhancements in the COSMOS optical data base (derived from digital scans of the UK Schmidt survey plates by COSMOS at the Royal Observatory Edinburgh (MacGillivray \& Stobie 1984; Heydon-Dumbleton et al. 1989). The resulting candidate list was carefully screened based on X-ray and optical information, literature data, and results from the optical follow-up observation program. The selection process was designed to provide a completeness in the final cluster catalogue in excess of $90 \%$ with respect to the flux-limited sample of GCA selected RASS sources. This high completeness of the cluster identification of the RASS sources ensures that the selection effects introduced by the optical identification process are minimized and negligible for our purpose (see also the statistics given in Paper I). Further tests provide support that this value of $>90 \%$ also describes the overall detection completeness of the flux-limited cluster sample in the survey area. For example an independent search for X-ray emission from the clusters catalogued by Abell et al. (1989) returns only one supplementary cluster, S567, with a flux above the flux-limit that has not been included in the REFLEX sample. In addition tests based on the Galactic latitude, redshift and photon count distribution, and on an independent screening of all significantly extended RASS X-ray sources in the survey region, are consistent with this claim (Böhringer et al. 2001a; Schuecker et al. 2001). Based on the X-ray spectral properties of the REFLEX cluster sources we can also estimate that at most $9 \%$ of the X-ray cluster sources may have a strong X-ray flux contribution from AGN (see Paper I).

To test further the identification of cluster candidates prior to the follow-up observations in La Silla, we adopted a very conservative scheme, as described in more detail in Paper I. To reject a source, we required it to have at least two (in some combinations three) properties which are incompatible with a cluster identification. The properties were drawn from the following list: X-ray source is point-like, X-ray source is too soft, no optical cluster visible on digitized optical images, known optical or radio AGN at the center of the X-ray emission.
This conservative scheme made the follow-up observations somewhat more expensive, but ensured a high completeness of the final catalogue.

The final cluster sample comprises 447 objects. The distribution of these sources in the sky is shown in Figs. 1 and 2.

\section{Determination of the $\mathrm{X}$-ray parameters}

The X-ray count rates, fluxes, and luminosities of the REFLEX clusters are determined from the count rate measurements provided by the GCA (Böhringer et al. 2000). In the first step the source count rates are integrated out to the radius where an effectively flat plateau of the cumulative source count curve is reached. The radius at which the plateau is reached is documented.

To determine the cluster X-ray flux we convert the measured count rate into an unabsorbed "nominal" X-ray flux for the ROSAT band ( 0.1 to $2.4 \mathrm{keV}), F_{\mathrm{n}}$, by assuming a RaymondSmith type spectrum (Raymond \& Smith 1977) for a temperature of $5 \mathrm{keV}$, a metallicity $\alpha$ of 0.3 of the solar value (Anders \& Grevesse 1989), a redshift of zero, and an interstellar hydrogen column density given for the line-of-sight in the compilation by Dickey \& Lockman (1990), as provided within EXSAS (Zimmermann et al. 1994). The value of $F_{\mathrm{n}}$ is used to make the flux cut independent of any redshift information (since the redshift is not available for all objects at the start of the survey). With the redshift value at hand, the unabsorbed X-ray flux is redetermined $\left(F_{\mathrm{x}}\right)$ with an improved spectral model, where the temperature is now estimated (iteratively) from the preliminarily derived X-ray luminosity and the luminosity-temperature relation (uncorrected for cooling flow effects) derived by Markevitch (1998). The estimated temperature of the cluster is now taken into account, by folding the appropriate thermal spectrum with the instrument response and the interstellar absorption, leading to a revised flux, $F_{\mathrm{x}}$ of the source (this correction is less than $5 \%$ for sources with an X-ray luminosity above $4 \times 10^{43} \mathrm{erg} \mathrm{s}^{-1}$ ). To obtain the cluster restframe luminosity from the flux, $F_{\mathrm{x}}$ we use the usual conversion with the cosmological luminosity distance and further scale the luminosity by the ratio of the luminosity integrated in the observed, redshifted and rest frame $0.1-2.4 \mathrm{keV}$ band. The latter is equivalent to the $\mathrm{K}$-correction. We note one simplification made in the above transformation. In calculating the revised flux, $F_{\mathrm{x}}$, we should have additionally taken the redshift effect into account by folding a redshifted source spectrum with the instrument response matrix and interstellar absorption effect to calculate the exact countrate-to-flux conversion factor. We found, however, that this correction is largest for the low temperature objects at high redshift and since we observe these objects only at closer distances, we established that this effect never becomes larger than $2 \%$ for any source in our catalogue and therefore we neglected this effect for the present analysis. In the various applications of our project we have used the results in Tables 2 and 3 for all the necessary conversion factors. Therefore in neglecting this effect we limit the necessary interpolations between observed and tabulated values to two-dimensional interpolations. This helps in the theoretical modeling when large parameter grids have to be evaluated. 


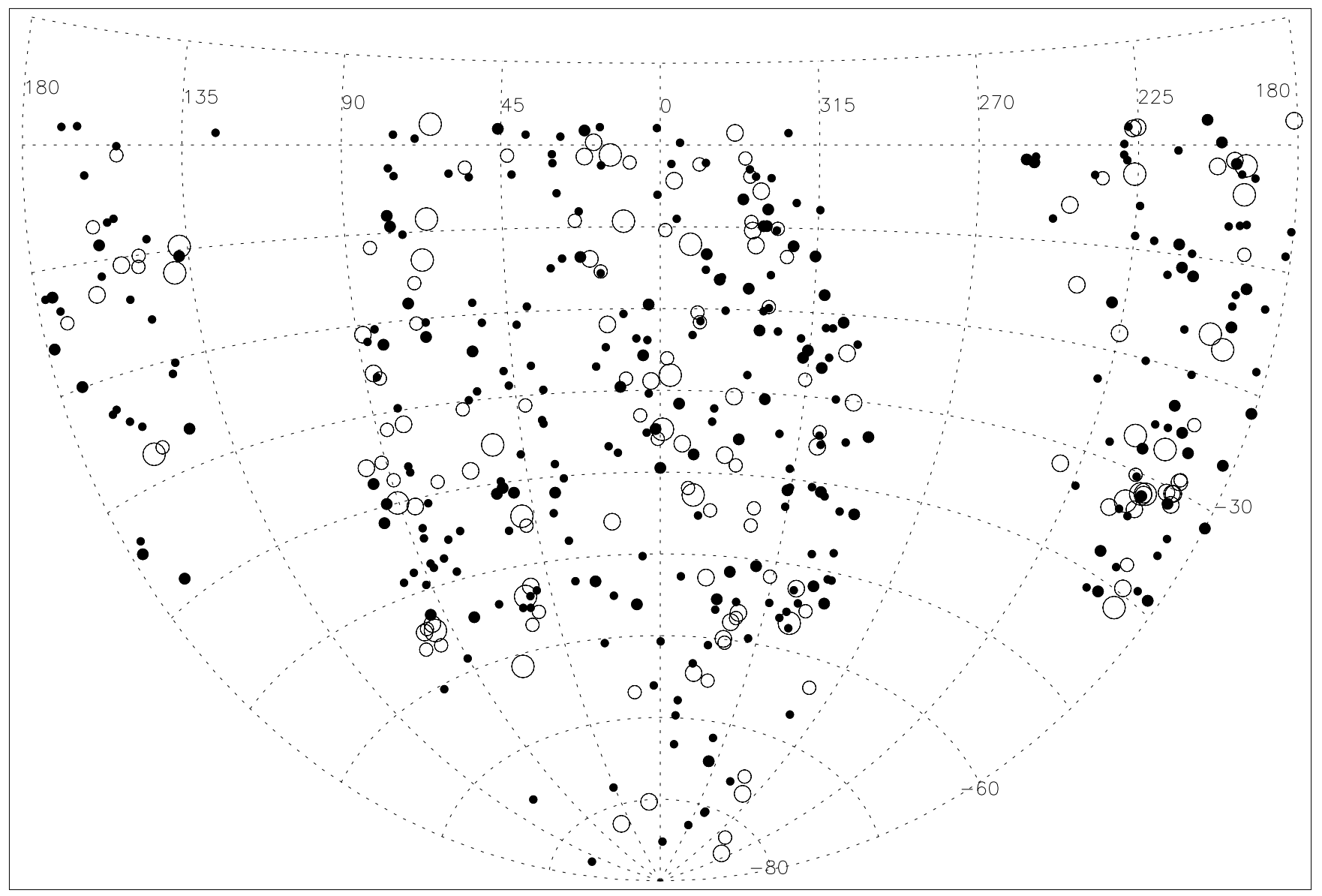

Fig. 1. Sky distribution in $\alpha$ and $\delta$ of the galaxy clusters in the REFLEX sample. The symbols give an indication of the cluster flux. The clusters are sorted into five flux bins: $3-5 \times 10^{-12}, 5-7 \times 10^{-12}, 7-10 \times 10^{-12}, 1-2 \times 10^{-11}$, and $\geq 2 \times 10^{-11} \mathrm{erg} \mathrm{s}^{-1} \mathrm{~cm}^{-2}$ and indicated by increasing symbol size, respectively. The three largest symbol classes are shown as open circles to avoid shading of other clusters.

Table 2. Count rate to flux conversion factors for different temperatures (for $Z=0.3$ solar, $z=0$ ) as a function of column density. The values quoted give the $0.1-2.4 \mathrm{keV}$ flux per counts in the 0.5 to $2 \mathrm{keV}$ band (channel 52 to 201 ) in units of $10^{-12} \mathrm{erg} \mathrm{s}^{-1} \mathrm{~cm}^{-2}$ counts ${ }^{-1}$. The last column gives the hardness ratio for an assumed temperature of $5 \mathrm{keV}$, defined as (counts $(0.5-2.0 \mathrm{keV})-$ counts $(0.1-0.4 \mathrm{keV})) /(\operatorname{counts}(0.5-2 \mathrm{keV})+$ counts $(0.1-0.4 \mathrm{keV}))$.

\begin{tabular}{|c|c|c|c|c|c|c|c|c|c|c|c|c|c|}
\hline \multirow[t]{2}{*}{$N_{\mathrm{H}}$} & \multicolumn{12}{|c|}{ Temperature } & \multirow{2}{*}{$\begin{array}{l}\mathrm{HR} \\
\text { for }\end{array}$} \\
\hline & 0.5 & 1.0 & 1.5 & 2.0 & 3.0 & 4.0 & 5.0 & 6.0 & 7.0 & 8.0 & 9.0 & 10.0 & \\
\hline $10^{20} \mathrm{~cm}^{-2}$ & $\mathrm{keV}$ & $\mathrm{keV}$ & $\mathrm{keV}$ & $\mathrm{keV}$ & $\mathrm{keV}$ & $\mathrm{keV}$ & $\mathrm{keV}$ & $\mathrm{keV}$ & $\mathrm{keV}$ & $\mathrm{keV}$ & $\mathrm{keV}$ & $\mathrm{keV}$ & $5 \mathrm{keV}$ \\
\hline 0.10 & 1.281 & 1.413 & 1.751 & 1.831 & 1.868 & 1.880 & 1.887 & 1.893 & 1.897 & 1.900 & 1.901 & 1.902 & 0.003 \\
\hline 0.30 & 1.291 & 1.422 & 1.761 & 1.842 & 1.879 & 1.891 & 1.898 & 1.904 & 1.908 & 1.910 & 1.912 & 1.913 & 0.089 \\
\hline 1.00 & 1.325 & 1.453 & 1.796 & 1.880 & 1.917 & 1.929 & 1.936 & 1.942 & 1.946 & 1.948 & 1.950 & 1.951 & 0.323 \\
\hline 3.02 & 1.429 & 1.543 & 1.900 & 1.989 & 2.028 & 2.040 & 2.046 & 2.052 & 2.055 & 2.057 & 2.059 & 2.059 & 0.691 \\
\hline 10.00 & 1.840 & 1.886 & 2.278 & 2.383 & 2.427 & 2.438 & 2.442 & 2.446 & 2.449 & 2.450 & 2.451 & 2.450 & 0.943 \\
\hline 30.20 & 3.654 & 3.213 & 3.583 & 3.718 & 3.766 & 3.766 & 3.762 & 3.760 & 3.756 & 3.753 & 3.749 & 3.744 & 0.978 \\
\hline
\end{tabular}

An extended version of this table is given in electronic form at CDS and our home page.

Thus, luminosities are calculated for the rest frame energy band 0.1 to $2.4 \mathrm{keV}$. We also account for the X-ray flux missed outside the detection aperture by the following correction. We correct the fluxes and luminosities based on a self-similar cluster model as described in Böhringer et al. (2000): a $\beta$-model (Cavaliere \& Fusco-Femiano 1976) with a $\beta$-value of $2 / 3$, a core radius that scales with mass, and an assumed extent of the X-ray halo out to 12 times the core radius. The correction procedure has been successfully tested by simulations based on the same cluster model. The typical mean correction factor is about $8 \%$ with the largest corrections of up to $30 \%$ occuring for the nearby groups which are extended and have a low surface brightness (Böhringer et al. 2000). Note that in contrast to our convention of extrapolating the corrected total flux out to the estimated virial radius, Ebeling et al. $(1996,1998)$ and De Grandi et al. (1999) extrapolate their flux corrections to 


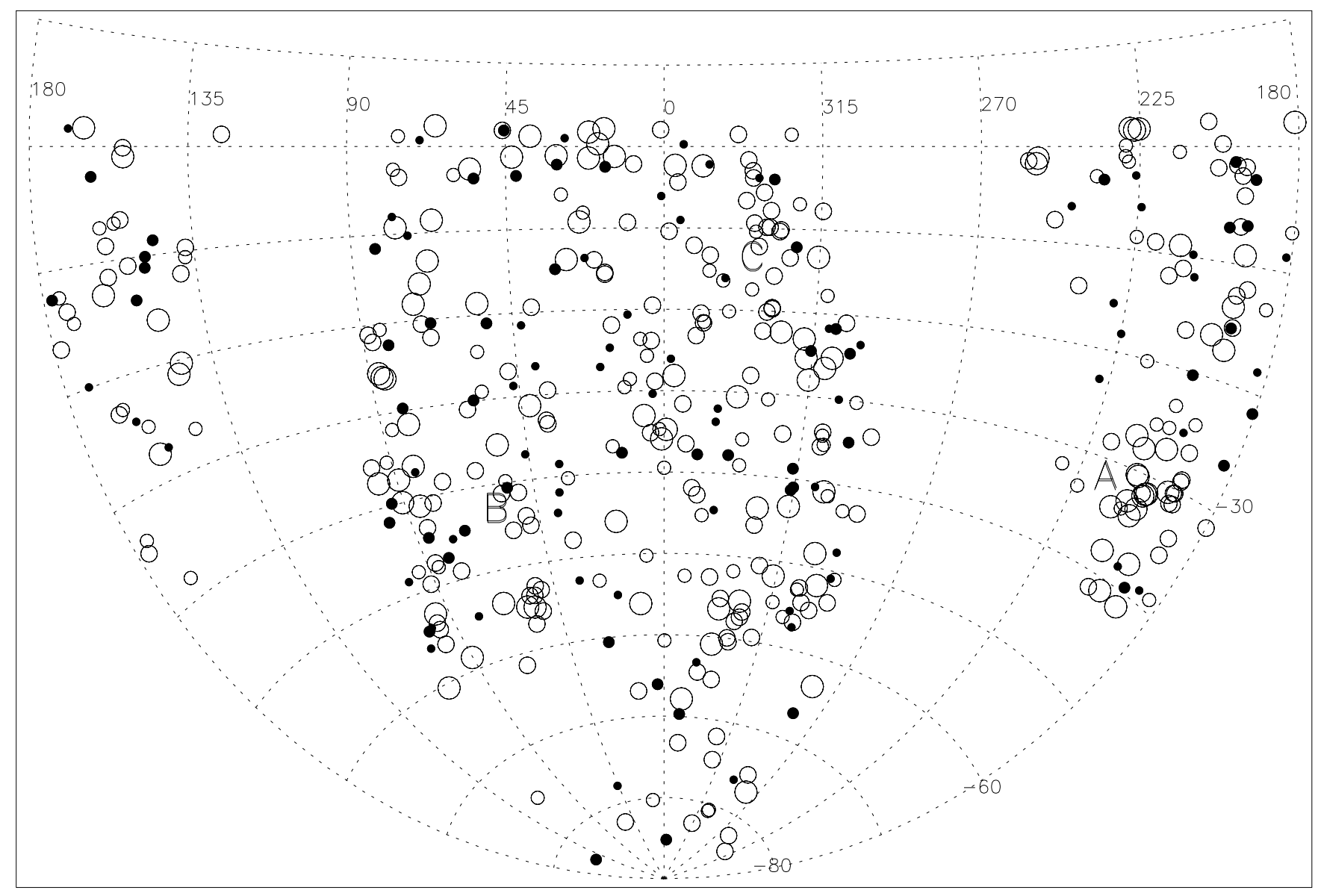

Fig. 2. The same sky distribution of REFLEX clusters as shown in Fig. 2, but now the symbols indicate the cluster distance. The clusters are sorted into five redshift classes: $z=0-0.05, z=0.05-0.1, z=0.1-0.15, z=0.15-0.2$, and $z \geq 0.2$ indicated by decreasing symbol size with increasing redshift. The three largest symbol classes are shown as open circles to avoid shading of other clusters. In this plot superstructures can be recognized. Three of the most prominent superclusters have been marked: A is the Shapley concentration partly overlapping with Hydra-Centaurus in the foreground, B is the Horologium-Reticulum complex, and C refers to the Aquarius-Cetus and Aquarius-Capricornus superclusters. For more details on these superstructures see Einasto et al. (2001).

Table 3. K-correction table for different temperatures and redshifts. The given value is to be multiplied with the luminosity in the observed band to obtain the luminosity in the rest frame band.

\begin{tabular}{|c|c|c|c|c|c|c|c|c|c|c|c|c|}
\hline \multirow[t]{3}{*}{ Rredshift } & \multicolumn{12}{|c|}{ Temperature } \\
\hline & 0.5 & 1.0 & 1.5 & 2.0 & 3.0 & 4.0 & 5.0 & 6.0 & 7.0 & 8.0 & 9.0 & 10.0 \\
\hline & $\mathrm{keV}$ & $\mathrm{keV}$ & $\mathrm{keV}$ & $\mathrm{keV}$ & $\mathrm{keV}$ & $\mathrm{keV}$ & $\mathrm{keV}$ & $\mathrm{keV}$ & $\mathrm{keV}$ & $\mathrm{keV}$ & $\mathrm{keV}$ & $\mathrm{keV}$ \\
\hline 0.0500 & 1.0026 & 0.9935 & 0.9867 & 0.9838 & 0.9800 & 0.9771 & 0.9750 & 0.9733 & 0.9720 & 0.9709 & 0.9700 & 0.9693 \\
\hline 0.1000 & 1.0086 & 1.0253 & 0.9852 & 0.9700 & 0.9596 & 0.9540 & 0.9502 & 0.9472 & 0.9449 & 0.9431 & 0.9415 & 0.9402 \\
\hline 0.1500 & 1.0126 & 1.0258 & 0.9806 & 0.9611 & 0.9450 & 0.9359 & 0.9299 & 0.9253 & 0.9217 & 0.9189 & 0.9166 & 0.9147 \\
\hline 0.2000 & 1.0273 & 1.0416 & 0.9771 & 0.9528 & 0.9314 & 0.9192 & 0.9112 & 0.9050 & 0.9003 & 0.8966 & 0.8936 & 0.8911 \\
\hline 0.2500 & 1.0452 & 1.0799 & 0.9880 & 0.9489 & 0.9197 & 0.9041 & 0.8940 & 0.8864 & 0.8806 & 0.8760 & 0.8724 & 0.8693 \\
\hline 0.3000 & 1.0497 & 1.0820 & 0.9833 & 0.9401 & 0.9070 & 0.8891 & 0.8775 & 0.8686 & 0.8619 & 0.8566 & 0.8523 & 0.8488 \\
\hline 0.4000 & 1.0584 & 1.0850 & 0.9768 & 0.9254 & 0.8837 & 0.8614 & 0.8469 & 0.8359 & 0.8276 & 0.8211 & 0.8159 & 0.8115 \\
\hline
\end{tabular}

An extended version of this table is given in electronic form at CDS and our home page.

infinity. The difference between the two approaches - which uses the same $\beta$-model with a slope of $2 / 3-$ is $8.3 \%$. Nevertheless the agreement between the results of, for example, Ebeling et al. (1998) and the results of Böhringer et al. (2000) have been found to show a bias smaller than this difference. We attribute this to the fact that the GCA method is capturing slightly more of the cluster flux than the other methods.

To allow the reader to easily and fully reproduce our present results and the results on the cosmological implications of 
Table 4. Conversion factor to be multiplied to the flux in the 0.5 to $2.0 \mathrm{keV}$ band to obtain the flux in the 0.1 to $2.4 \mathrm{keV}$ band as a function of temperature.

\begin{tabular}{ll}
\hline \hline $\begin{array}{l}\text { Temperature } \\
(\mathrm{keV})\end{array}$ & $\begin{array}{l}\text { Flux } \\
\text { ratio }\end{array}$ \\
\hline 0.5 & 1.387 \\
1.0 & 1.496 \\
1.5 & 1.615 \\
2.0 & 1.623 \\
3.0 & 1.618 \\
4.0 & 1.611 \\
5.0 & 1.607 \\
6.0 & 1.605 \\
7.0 & 1.603 \\
8.0 & 1.599 \\
9.0 & 1.598 \\
10.0 & 1.597 \\
\hline
\end{tabular}

the REFLEX project published in other papers of this series, without having to resort to ROSAT instrument specific calculations, we provide here all the conversion tables used in the flux and luminosity determination described above. In the underlaying calculations we were aiming for an accuracy of better than about $2-3 \%$ (e.g. in the energy dependent vignetting correction, the tested difference between calculations with different radiation codes), so that the errors from the conversion factors is in any case negligible compared to the measurement errors. Table 2 gives the conversion factor from the observed count rate in the ROSAT hard band (defined by the range from energy channel 52 to 201) to the flux in the ROSAT band (0.1-2.4 keV) for various temperatures covering the relevant temperature range, $\alpha=0.3$ solar, and $z=0$, as a function of the interstellar column density. A graphical representation of this function for three selected temperatures can be found in Böhringer et al. (2000, Fig. 8a) together with the column density dependence of the hardness ratio for the same temperatures (Fig. 8b). This table is used to interpolate the count rate conversion factor for the first step described above, where a fixed temperature of $5 \mathrm{keV}$ is used. In the second step the calculation is improved by interpolating to the estimated temperature. Table 3 then provides the K-correction factors to convert the redshifted luminosity measure into rest-frame luminosity as a function of redshift and temperature of the cluster.

For an easy comparison of the catalogue with data given in other frequently used energy bands, we provide in Table 4 the conversion factor from the $0.1-2.4 \mathrm{keV}$ to the $0.5-2.0 \mathrm{keV}$ band as a function of temperature. Table 5 provides the conversion from the ROSAT band $(0.1-2.4 \mathrm{keV})$ to the bolometric system. The print version of the paper gives only a few example lines for Tables 2 through 5. The full tables can be obtained from the electronic version of the paper.

\section{Redshift determination}

For cluster candidates with published redshifts we adopted the following procedure to obtain the final redshift. The search for
Table 5. Conversion factor of the flux/luminosity in the 0.1 to $2.4 \mathrm{keV}$ band to bolometric flux/luminosity as a function of temperature.

\begin{tabular}{ll}
\hline \hline $\begin{array}{l}\text { Temperature } \\
(\mathrm{keV})\end{array}$ & $\begin{array}{l}\text { Flux } \\
\text { ratio }\end{array}$ \\
\hline 0.30 & 1.443 \\
0.51 & 1.187 \\
1.02 & 1.269 \\
1.98 & 1.448 \\
3.00 & 1.660 \\
5.10 & 2.122 \\
8.08 & 2.743 \\
10.17 & 3.152 \\
15.04 & 4.053 \\
\hline
\end{tabular}

An extended version of this table is given in electronic form at CDS and our home page.

redshift information in the literature was conducted with an aperture radius of 7 arcmin for more distant clusters and the search radius was increased to half the virial radius (half the virial radius was taken to be 5 times the core radius estimated from $L_{\mathrm{X}}$ as described in Böhringer et al. 2000) if this radius was larger than 7 arcmin. This is of course an iterative process in which a first redshift result was chosen for which the search radius was calculated and then a refined search for cluster galaxy members was performed in NED. This was found to be very important for the nearby clusters where an increasing number of redshifts becomes available from large redshift surveys which help greatly in improving the cluster redshifts. The final redshift taken was the cluster redshift that involved the largest number of galaxy redshifts. If no reliable cluster redshift was available, the cluster redshift was computed from the median of individual galaxy redshifts located within the aperture radius around the central target position after the rejection of obvious non-members of the cluster (see below). The secondary line-of-sight clustering found for a number of REFLEX clusters is further described in Sect. 7. The present literature redshift compilation is largely based on information requested from NED in July 2003.

Cluster candidates without published redshifts or reliable identification were observed from 1992 to 1999 within a large ESO key programme (Böhringer et al. 1998; Guzzo et al. 1999). For a detailed spectroscopic follow-up we used for the brighter objects the ESO $1.5 \mathrm{~m}$ with the Boller \& Chivens spectrograph and the ESO $2.2 \mathrm{~m}$ with the EFOSC-2 instrument. For the more distant and thus fainter objects the ESO $3.6 \mathrm{~m}$ telescope with the EFOSC-1 (later EFOSC-2) instrument was used. This instrument was preferentially used in multi-object spectroscopic mode, but also long-slit spectra were taken with EFOSC for some of the clusters. If necessary we also performed CCD imaging in order to test for galaxy overdensities in the target direction. A detailed description of the observing strategy, the reduction of the spectra, redshift measurements, astrometry, and morphological classification is in preparation (Guzzo et al. 2004, in preparation). A brief summary is given below. 


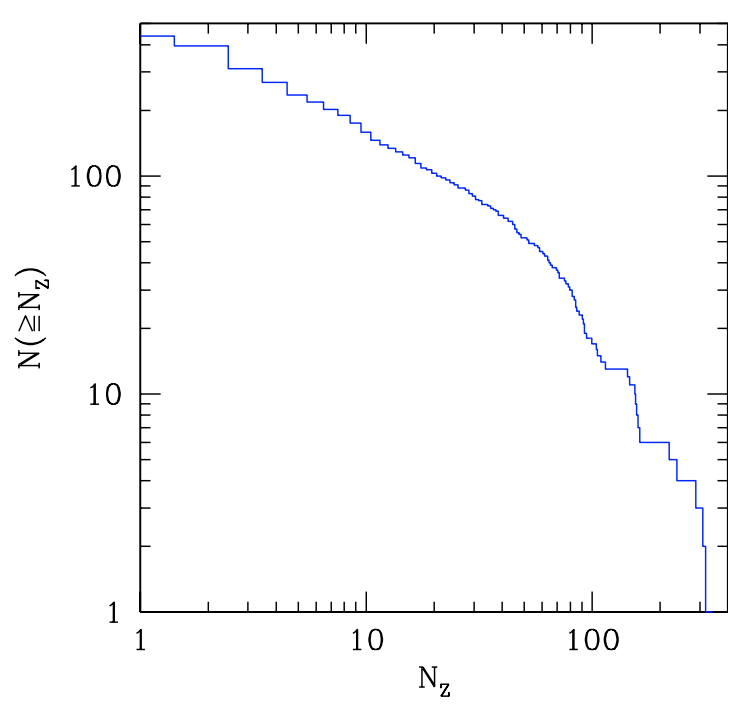

Fig. 3. Cumulative distribution, $N_{\mathrm{cl}}\left(\geq N_{z}\right)$, of the number of galaxy redshifts known for the clusters in the REFLEX sample.

The main goal of the follow-up observations was to get for each X-ray cluster candidate at least five spectra, which we found was enough for a first identification of the X-ray source and for a good estimate of the cluster redshift. This comparatively small number of spectra results from the fact that the optical follow-up could be restricted to the location of the X-ray peak, that is, to the region of the expected deepest cluster potential well where contamination by background galaxies is minimal. In order to optimize the efficiency of cluster identification and redshift measurement, for clusters with a few bright (nearby) galaxies, spectra were observed in the single-slit mode, whereas for clusters with many faint galaxies the multi-slit mode was chosen. The multi-slit mode gives spectra for about 10-25 galaxies, so that for many clusters we also have information about the velocity dispersion (Ortiz-Gil et al. 2004).

Figure 3 shows the cumulative distribution of the REFLEX clusters with a number of member galaxy redshifts greater than the given limit. Indeed about half of the clusters have 5 or more galaxy redshifts and the derived redshift is fairly secure. However, about 42 clusters feature only one galaxy redshift, mostly values coming from the literature. The available observation time budget for this project did not allow for a redetermination of all the literature values. Still most of these data yield a reliable, as they refer to the central brightest cluster galaxy at the center of the X-ray emission. For 8 clusters in the catalogue we were so far not able to find the information in the literature on the number of galaxies from which the cluster redshift was determined.

The majority of spectra were reduced in a standard manner (bias and flat field correction, wavelength calibration) using procedures from the IRAF software package. Cosmics were rejected from the combination of at least two spectral exposures obtained for each spectral observation. All spectra were visually classified into the categories elliptical galaxy, spiral galaxy, Seyfert 1/2 galaxy, quasar, starforming galaxy (tentatively), and stellar. Heliocentric redshifts were determined from cross-correlation with a sample of 17 template spectra with spectral types covering the large range usually found in clusters of galaxies and in the field. In addition, redshifts were also determined from emission lines (if present), including a measurement of their equivalent widths. The final redshifts of the clusters were obtained from the median of the measured galaxy redshifts after the rejection of obvious non-members. The criterion for the assignment of the cluster membership was a maximum velocity deviation of $3000 \mathrm{~km} \mathrm{~s}^{-1}$ from the median.

All optical and X-ray information is collected and organized in a data base which we are planing to make publicly available. A more detailed account of the optical data will be given in a forthcoming paper listing in particular the individual galaxy redshifts determined in this ESO key program (Guzzo et al.)

\section{The catalogue}

Table 6 list the X-ray properties and redshifts of the 447 galaxy clusters of the REFLEX sample (compared to the previously used sample of 449 clusters, 7 clusters have been removed as detailed in the Appendix, 4 clusters considered before as being contaminated by non-cluster emission have been added, and one double cluster has been split into two sources). The columns of the table provide: (1) the REFLEX name; (2) a previous catalogue name, where for some of the smaller galaxy groups the name refers to the central dominant galaxy in the group (e.g. the NGC name of this galaxy); (3) and (4) the right ascension and declination for the epoch J2000 in hours (degrees), minutes, and seconds; (5) the redshift; (6) the number of galaxies with which the redshift has been determined after the rejection of non-members (a zero means that no information is available about the number of galaxies used to determine the cluster redshift); (7) and (8) the measured, unabsorbed X-ray flux, $F_{\mathrm{x}}$, in units of $10^{-12} \mathrm{erg} \mathrm{s}^{-1} \mathrm{~cm}^{-2}$ for the $0.1-2.4 \mathrm{keV}$ energy band and the fractional error in percent (note that this is not the nominal flux, $F_{\mathrm{n}}$, used to define the flux limit of REFLEX; $F_{\mathrm{n}}$ is given in Table 7); (9) the X-ray luminosity uncorrected for missing flux in units of $10^{44} \mathrm{erg} / \mathrm{s}$ in the rest frame 0.1 to $2.4 \mathrm{keV}$ band; (10) the aperture radius in arcmin within which the X-ray count rate and flux were determined (the radius where the plateau value is reached in the cumulative count rate curve of the GCA); (11) the 0.1-2.4 keV luminosity corrected for the estimated flux lost outside the measurement aperture; (12) the interstellar column density in units of $10^{20} \mathrm{~cm}^{-2}$ as obtained from the $21 \mathrm{~cm}$ observations by Dickey \& Lockman (1990) and Stark et al. (1992); (13) comment flags as described below; and (14) references to literature values for the cluster redshifts. The comment flags refer to: (L) clusters which are too extended for the standard GCA count rate determination in $2 \times 2$ degree $^{2}$ fields, and therefore were analysed in $4 \times 4$ or $8 \times 8$ degree $^{2}$ fields; (B) clusters blended with point sources or double clusters which were deblended; (X) clusters where the details of the source identification are commented in the Appendix; (P) parts of close cluster pairs or groups; and (D) double clusters discussed in Sect. 7. For 12 objects marked with an astrisk in Col. (13) the cluster origin of the X-ray emission is not completely certain as detailed in the Appendix. 
Table 6. The REFLEX cluster catalogue.

\begin{tabular}{|c|c|c|c|c|c|c|c|c|c|c|c|c|c|}
\hline $\begin{array}{l}\text { Name } \\
\text { (1) }\end{array}$ & $\begin{array}{l}\text { Alt. name } \\
\text { (2) }\end{array}$ & $\begin{array}{r}\mathrm{RA}(2000) \\
\text { (3) }\end{array}$ & $\begin{array}{r}\operatorname{Dec}(2000) \\
(4)\end{array}$ & $\begin{array}{r}z \\
(5)\end{array}$ & $\begin{array}{r}N_{\text {gal }} \\
(6)\end{array}$ & $\begin{array}{l}F_{\mathrm{x}} \\
(7)\end{array}$ & $\begin{array}{r}\text { Err. } \\
(8)\end{array}$ & $\begin{array}{l}L_{\mathrm{x}} \\
\text { (9) }\end{array}$ & $\begin{array}{r}R_{\text {ap }} \\
(10)\end{array}$ & $\begin{array}{c}L_{\mathrm{x}}{ }^{*} \\
(11)\end{array}$ & $\begin{array}{r}N_{\mathrm{H}} \\
(12)\end{array}$ & $\begin{array}{r}\mathrm{Cm} \\
(13)\end{array}$ & $\begin{array}{l}\text { Ref. } \\
\text { (14) }\end{array}$ \\
\hline RXCJ0003.1-0605 & A2697 & 000311.8 & -060510 & 0.2320 & 0 & 4.497 & 12.4 & 6.395 & 7.5 & 6.876 & 3.1 & & 139 \\
\hline RXCJ0003.2-3555 & A2717 & 000312.1 & -355538 & 0.0490 & 40 & 7.537 & 17.8 & 0.421 & 10.0 & 0.478 & 1.1 & & 2 \\
\hline RXCJ0003.8+0203 & A2700 & 000350.6 & +020348 & 0.0924 & 9 & 4.155 & 18.8 & 0.855 & 8.5 & 0.929 & 3.0 & & S, 10 \\
\hline RXCJ0006.0-3443 & A2721 & 000603.0 & -344327 & 0.1147 & 75 & 5.832 & 13.6 & 1.875 & 10.0 & 1.995 & 1.2 & & 1 \\
\hline RXCJ0011.3-2851 & A2734 & 001120.7 & -285118 & 0.0620 & 83 & 12.014 & 9.0 & 1.089 & 12.5 & 1.197 & 1.8 & & 2 \\
\hline RXCJ0013.6-1930 & A0013 & 001338.3 & -193008 & 0.0940 & 37 & 6.071 & 11.3 & 1.285 & 11.5 & 1.353 & 2.0 & & $\mathrm{~S}, 2,140,141$ \\
\hline RXCJ0014.3-6604 & A2746 & 001418.4 & -660439 & 0.1599 & 5 & 4.485 & 13.8 & 2.907 & 7.5 & 3.160 & 2.8 & $\mathrm{X}$ & $\mathrm{E}$ \\
\hline RXCJ0014.3-3023 & A2744 & 001418.8 & -302300 & 0.3066 & 65 & 4.964 & 18.7 & 12.787 & 12.0 & 12.916 & 1.6 & & 142,143 \\
\hline RXCJ0015.4-2350 & & 001524.0 & -235042 & 0.0645 & 4 & 3.426 & 18.3 & 0.336 & 11.5 & 0.354 & 2.5 & $\mathrm{X}$ & 33 \\
\hline RXCJ0017.5-3509 & A2755 & 001733.7 & -350954 & 0.0968 & 23 & 3.211 & 20.0 & 0.729 & 11.5 & 0.752 & 1.3 & & E, $3,33,140,141$ \\
\hline RXCJ0020.7-2542 & A0022 & 002042.8 & -254237 & 0.1410 & 3 & 5.910 & 12.1 & 2.909 & 7.5 & 3.232 & 2.3 & & 1 \\
\hline RXCJ0025.5-3302 & S0041 & 002532.4 & -330250 & 0.0491 & 3 & 8.818 & 9.7 & 0.494 & 13.0 & 0.537 & 1.7 & & 26,144 \\
\hline RXCJ0027.3-5015 & A2777 & 002721.3 & -501504 & 0.1448 & 1 & 4.286 & 17.1 & 2.247 & 8.5 & 2.390 & 1.7 & & E, 145 \\
\hline RXCJ0028.6-2338 & A0042 & 002839.3 & -233814 & 0.1120 & 5 & 4.836 & 13.3 & 1.491 & 13.0 & 1.521 & 1.8 & & 33 \\
\hline RXCJ0034.6-0208 & & 003436.0 & -020824 & 0.0812 & 2 & 8.629 & 7.9 & 1.360 & 28.0 & 1.388 & 2.8 & DL & S, 139 \\
\hline
\end{tabular}

${ }^{a}$ C10053-37; ${ }^{b}$ cluster from the catalogue of Wegner et al. $(1996,1999) ;{ }^{c}$ from the ROSAT Bright Source Catalogue

(Fischer et al. 1998; Schwope et al. 2000); Key to the redshift references: (E, H) ESO-Key Programme; (S) South Galactic Pole Project (part of Ph.D. Thesis K.A. Romer); (1) Struble \& Rood (1999); (2) Katgert et al. (1996); (3) Katgert et al. (1998); (4) Postman \& Lauer (1995); (5) Lauberts \& Valentijn (1989); (6) Ebeling et al. (1996); (7) Bauer et al. (2000); (8) Kapahi et al. (1998); (9) Abell et al. (1989); (10) Slinglend et al. (1998); (11) Müller et al. (1999); (12) Smith et al. (2000); (13) Solanes \& Stein (1998); (14) Stein (1996); (15) NED information prior to 1992 without reference; (16) Tucker et al. (2000); (17) Dalton et al. (1994); (18) Dale et al. (1998); (19) Quintana \& Ramirez (1994); (20) Da Costa et al. (1998); (21) Patten et al. (2000); (22) Barton et al. (1996); (23) Mahdavi et al. (2000); (24) Loveday et al. (1996); (25) Garilli et al. (1993); (26) Ratcliffe et al. (1998); (27) De Grandi et al. (1999); (28) Green et al. (1998); (29) Dalton et al. (1997); (30) Muriel et al. (1995), and 151 further references given in the electronic version of the table.

The complete table is given in the electronic version of the paper, in the astro-ph/version and at our home page.

Further more detailed X-ray properties on the REFLEX clusters as determined with the GCA method are given in Table 7, where the following columns are listed: (1) name; (2) and (3) repeat the J2000 sky coordinates, but now in units of decimal degrees; (4) the count rate as measured with the GCA method for the aperture size given in Col. 10 in Table 6; (5) X-ray flux, $F_{\mathrm{n}}$, determined in the first step for an assumed temperature of $5 \mathrm{keV}$ used for the source selection; (6) the X-ray luminosity in the rest frame 0.1 to $2.4 \mathrm{keV}$ band uncorrected for missing (unabsorbed) flux; (7) the number of source photons detected within the reference aperture (Col. 14); (8) the probability for the X-ray source to be a point source in values of $-\log _{10}(P)$ (determined as described below); (9) and (10) the best fitted core radius for the $\beta$-model fit and a minimal core radius still consistent within $2 \sigma$ error limits, respectively. Note that the core radii determined here are only a qualitative measure for the source extent, since the errors are very large and the fitting grid was coarsely spaced. Therefore core radii and their lower limiting values can give a further qualitative feeling for the extent of the $\mathrm{X}$-ray sources, but we recommend not to use these results for $r_{\mathrm{c}}$ as a quantitative measure of cluster shapes at this point. Columns (11) and (12) give the spectral hardness ratio defined by the equation given below and its Poisson error. Column (13) indicates the deviation of the measured hardness ratio from the expectation value calculated for given $N_{\mathrm{H}}$ and for an assumed temperature of $5 \mathrm{keV}$ as factors of $\sigma$. The last Col. (14) repeats from Table 6 the aperture radius within which the source count rate was measured, but now in physical units of Mpc. This is the radius where the cumulative source count rate profile reaches the plateau value. The print version of this paper gives only a few example lines of this table. The full table as well as a second version of Table 6 for an Einstein-de Sitter Universe model is provided in the electronic version.

The probability of an X-ray source to be a point source, as given in Col. 8 was determined by means of a KolmogorovSmirnov test comparing the normalized radially cumulative photon distribution within an aperture radius of 6 arcmin with the expectations for a point source and background. In this test we consider a source to be significantly extended if the probability is less than $1 \%$, corresponding to an entry value in Table 7 larger than 2. The source hardness ratio, as given in Col. 11, was determined from the source count rate in the soft (ROSAT PSPC channel 11 to 40) and hard (PSPC channel 52 to 201) band by the formula:

$H R=\frac{H-S}{H+S}$

where $H$ is the hard band and $S$ the soft band count rate. 
Table 7. X-ray properties of the REFLEX clusters.

\begin{tabular}{|c|c|c|c|c|c|c|c|c|c|c|c|c|c|}
\hline Name & $\begin{array}{r}\text { RA } \\
(2000)\end{array}$ & $\begin{array}{r}\text { Dec } \\
(2000) \\
(3)\end{array}$ & $\begin{array}{c}\text { Count } \\
\text { rate } \\
\mathrm{s}^{-1} \\
(4)\end{array}$ & $\begin{array}{c}F_{\mathrm{n}} \\
10^{-12} \mathrm{erg} \\
\mathrm{s}^{-1} \mathrm{~cm}^{-2} \\
\quad(5)\end{array}$ & $\begin{array}{c}L_{\mathrm{x}} \\
10^{44} \\
\mathrm{erg} \mathrm{s}^{-1} \\
\quad(6)\end{array}$ & $N_{\mathrm{ph}}$ & $P_{\text {ext }}$ & $\begin{array}{r}\operatorname{arcmin} \\
(9)\end{array}$ & $\begin{array}{r}r_{\mathrm{c}}(\min ) \\
\operatorname{arcmin} \\
(10)\end{array}$ & (11) & $H R_{\mathrm{err}}$ & $\begin{array}{c}\Delta H R \\
(\sigma)\end{array}$ & $\begin{array}{r}\mathrm{Mpc} \\
(14)\end{array}$ \\
\hline RXCJ0003.1-0605 & 0.7991 & -6.0860 & 0.218 & 4.473 & 6.395 & 76.3 & 3.8 & 1.5 & 1.0 & 0.7 & 0.1 & 0.06 & 1.664 \\
\hline RXCJ0003.2-3555 & 0.8006 & -35.9271 & 0.400 & 7.762 & 0.421 & 56.8 & 7.5 & 1.5 & 0.5 & 0.5 & 0.2 & 0.70 & 0.575 \\
\hline RXCJ0003.8+0203 & 0.9607 & +2.0634 & 0.205 & 4.192 & 0.855 & 74.2 & 3.3 & 1.0 & 0.5 & 0.8 & 0.1 & 1.18 & 0.877 \\
\hline RXCJ0006.0-3443 & 1.5126 & -34.7241 & 0.301 & 5.853 & 1.875 & 68.0 & 5.4 & 1.5 & 1.0 & 0.2 & 0.1 & -1.05 & 1.248 \\
\hline RXCJ0011.3-2851 & 2.8364 & -28.8551 & 0.612 & 12.129 & 1.089 & 155.4 & 13.9 & 1.5 & 1.5 & 0.4 & 0.1 & -1.53 & 0.896 \\
\hline RXCJ0013.6-1930 & 3.4094 & -19.5021 & 0.306 & 6.089 & 1.285 & 95.8 & 6.1 & 1.5 & 1.5 & 0.5 & 0.1 & -0.61 & 1.203 \\
\hline RXCJ0014.3-6604 & 3.5768 & -66.0775 & 0.220 & 4.474 & 2.907 & 60.9 & 0.4 & 0.0 & 0.0 & 0.6 & 0.1 & -0.80 & 1.240 \\
\hline RXCJ0014.3-3023 & 3.5783 & -30.3834 & 0.250 & 4.929 & 12.787 & 30.5 & 1.3 & 0.5 & 0.0 & 0.2 & 0.2 & -1.67 & 3.255 \\
\hline RXCJ0015.4-2350 & 3.8500 & -23.8450 & 0.175 & 3.526 & 0.336 & 48.5 & 1.8 & 6.0 & 2.0 & 0.4 & 0.2 & -1.36 & 0.854 \\
\hline RXCJ0017.5-3509 & 4.3904 & -35.1650 & 0.166 & 3.241 & 0.729 & 58.8 & 6.7 & 3.0 & 2.0 & 0.3 & 0.2 & -0.73 & 1.236 \\
\hline
\end{tabular}

The complete table is given in electronic form at CDS and our home page.

Figure 1 shows the sky distribution of the REFLEX clusters with five different X-ray flux classes marked by different symbols. The empty region around $\mathrm{RA}=75 \mathrm{deg}(5 \mathrm{~h})$ and Dec $=-70 \mathrm{deg}$ is due to the excision of the regions of the Large and Small Magellanic Clouds. Figure 2 shows the same sky distribution but now with the clusters sorted in five different redshift classes. This illustrates the large scale clustering of the clusters, and several known superclusters are marked.

In the Appendix we give additional notes on some of the interesting and uncertain identifications.

\section{Selection properties of the cluster sample}

A number of tests in Papers I through III demonstrate the quality of the catalogue, in particular the completeness and homogeneity. For example through the $\log N-\log S$ distribution, a $V / V_{\max }$-test, and the photon number distribution, do not indicate a significant deficiency at faint fluxes or low source photon numbers. The sample shows a large scale density homogeneity at least out to $z=0.15$ with pronounced traces of large scale structure on scales smaller than the sample size (Paper III). The two-point correlation function (Paper II) shows a good overall isotropy (except for possibly small dynamical redshift distortion effects which are expected and currently investigated) and the number count distribution on large scales $\left(\geq 50 h^{-1} \mathrm{Mpc}\right)$ is approximately Gaussian (Paper IV). We do not see any significant bias in the cluster density as a function of Galactic latitude or longitude (Papers II and III). Here we provide another test, showing the cluster density as a function of interstellar absorption. Figure 4 shows the surface density of clusters averaged over $0.5 \times 10^{20} \mathrm{~cm}^{-2}$ column density intervals and compares it to the distribution of $N_{\mathrm{H}}$ over the sky. The density is almost constant within the formal Poissonian errors (which do not include fluctuations from large-scale structure and cosmic variance) up to the region where the statistics becomes too poor and the expectation value drops below one or two. Since the column density as well as the stellar density, which hampers

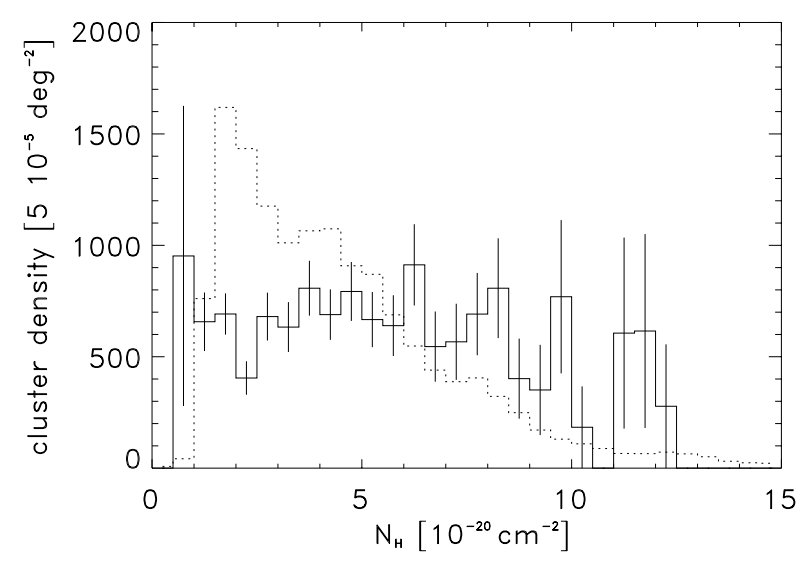

Fig. 4. Sky density of the REFLEX clusters as a function of the Galactic hydrogen column density (solid line) in units of $\mathrm{deg}^{-2}$ multiplied by a factor of 20000 . The dotted line gives the sky area distribution of $N_{\mathrm{H}}$ values in units of $\mathrm{deg}^{2}$ for $0.5 \times 10^{20} \mathrm{~cm}^{-2}$ bins.

the cluster identification, increases with decreasing $|b|$, we are also testing here the bias in the optical cluster identification due to crowding of the sky fields near the Galactic plane and find no major effect. These plots have to be compared with similar tests conducted with the optically identified clusters in the Abell catalogue (e.g., Ebeling et al. 1996).

Another mark of quality of the REFLEX cluster identification is the fact that the vast majority of the clusters, $79.5 \%$, have been identified as extended sources. Figure 5 shows the $\log N-\log S$ distribution of the subsample of clusters detected as significantly extended in the GCA.

To reproduce the observational results described here in theoretical modeling the precise selection function of the REFLEX survey has to be known. Two major factors modify the depth of our survey as a function of sky position: the RASS exposure time and the interstellar column density in the line-of-sight. We model the sensitivity function which depends on these two parameters in the following way: we calculate the flux limit reached per number of photons specified to be 


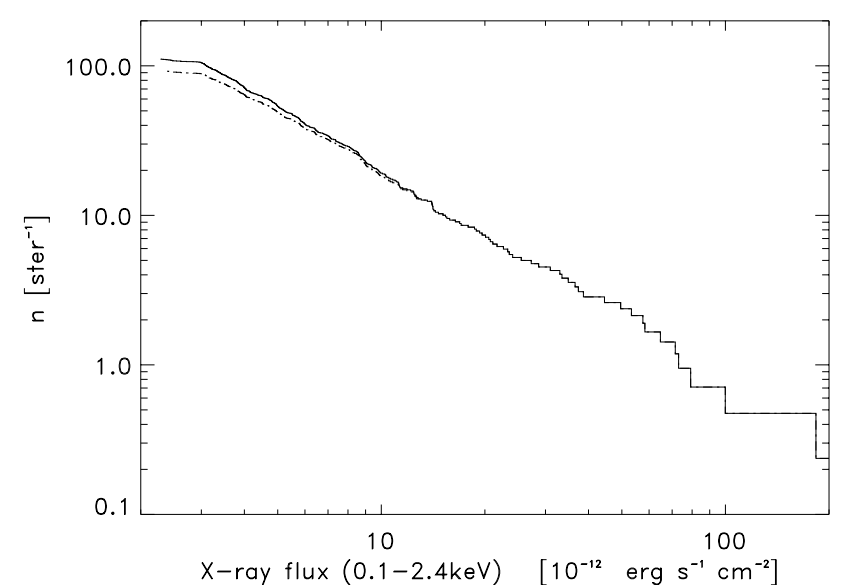

Fig. 5. $\log N-\log S$ distribution of the REFLEX clusters. The upper curve shows all the clusters while the lower, dashed curve shows the $79.5 \%$ fraction of significantly extended sources as measured with the KS test for the survey point spread function.

Table 8. Statistics of the sky coverage as a function of the sensitivity. A graphical representation of this table can be found in Böhringer et al. (2001, Fig. 23).

\begin{tabular}{ll}
\hline \hline $\begin{array}{l}\text { Sensitivity limit } \\
10^{-13} \mathrm{erg} \mathrm{s}^{-1} \mathrm{~cm}^{-2} \text { count }^{-1}\end{array}$ & $\begin{array}{l}\text { Fraction of the } \\
\text { survey area }\end{array}$ \\
\hline 0.102 & $8.6 \times 10^{-4}$ \\
0.300 & 0.032 \\
0.500 & 0.192 \\
0.700 & 0.574 \\
1.000 & 0.780 \\
2.000 & 0.940 \\
3.002 & 0.970 \\
5.000 & 0.984 \\
\hline
\end{tabular}

The complete table is given in electronic form at CDS and our home page.

required for secure source detection. In this sense Table 8 gives the sky area in the REFLEX survey for a given flux limit for the detection of one photon. This sky coverage table is for example used in the calculation of the luminosity function in Paper IV, where we assume conservatively that at least 30 photons are detected for the cluster. The flux limit as listed in the first column of Table 8 has therefore to be multiplied by 30 and we note for example that $78 \%$ of the survey area reaches the nominal flux limit of the REFLEX survey of $3 \times 10^{-12} \mathrm{erg} \mathrm{s}^{-1} \mathrm{~cm}^{-2}$. Note also, that this excludes some clusters in the catalogue with less than 30 source photon counts from the analysis. For the largescale structure analysis in Papers II and III we have included more clusters for a better three-dimensional coverage and relaxed the detection requirement to 10 source photons. In this case the REFLEX survey reaches the nominal flux limit in 97\% of the survey area, which corresponds to a more simple survey window function in $\mathrm{k}$-space for the determination of the density fluctuation power spectrum.

For the large-scale structure modeling, e.g. for the construction of the comparison Poisson sample used in the evaluation
Table 9. Sensitivity as a function of the sky position used to produce the sensitivity map shown in Böhringer et al. (2001, Fig. 22). Also given are the sky distribution of the RASS II exposure (shown in Böhringer et al. 2001, Fig. 2) and the interstellar column density distribution in the REFLEX area.

\begin{tabular}{lllll}
\hline \hline $\begin{array}{l}\text { RA } \\
(\mathrm{J} 2000)\end{array}$ & $\begin{array}{l}\text { Dec } \\
(\mathrm{J} 2000)\end{array}$ & $\begin{array}{l}\text { exposure } \\
(\mathrm{s})\end{array}$ & $\begin{array}{l}N_{\mathrm{H}} \\
10^{20} * \mathrm{~cm}^{-2}\end{array}$ & $\begin{array}{l}\text { Sensitivity limit } \\
10^{-13} \mathrm{erg} \mathrm{s}^{-1} \mathrm{~cm}^{-2} \\
\mathrm{count}^{-1}\end{array}$ \\
\hline 0.0000 & 2.0000 & 401.09 & 3.300 & 0.514 \\
1.0006 & 2.0000 & 360.85 & 3.000 & 0.567 \\
2.0012 & 2.0000 & 250.08 & 3.000 & 0.818 \\
3.0018 & 2.0000 & 332.16 & 3.040 & 0.616 \\
4.0024 & 2.0000 & 398.57 & 3.070 & 0.514 \\
5.0030 & 2.0000 & 404.37 & 3.150 & 0.508 \\
6.0037 & 2.0000 & 442.88 & 3.150 & 0.464 \\
7.0043 & 2.0000 & 662.26 & 3.040 & 0.309 \\
8.0049 & 2.0000 & 464.21 & 2.730 & 0.438 \\
9.0055 & 2.0000 & 291.09 & 2.660 & 0.696 \\
10.0061 & 2.0000 & 323.05 & 2.320 & 0.622 \\
\hline
\end{tabular}

The complete table is given in electronic form at CDS and our home page.

of the two-point correlation function or the density distribution power spectrum, the sensitivity limit as a function of the sky position has to be known. This has been constructed from the exposure map of the RASS II product and the $21 \mathrm{~cm}$ maps from Dickey \& Lockman (1990) and Stark et al. (1992), and is given in a similar way in Table 9 . In fact Table 8 is only a cumulative account of Table 9. The table gives the sensitivity distribution on a roughly one square degree grid. It lists also the exposure and interstellar column density distribution used in the calculation. For both tables only a few example lines are given in the print version of the paper, but the full tables (which in case of Table 9 consists of 13902 lines) are provided in the electronic version of the paper.

\section{Notes on double clusters}

One problem in defining an X-ray cluster catalogue, as well as in comparing different cluster catalogues, is the identification of double clusters and of single clusters with substructure. This becomes obvious when we compare our results to other compilations in the next section. For the present catalogue we have taken a very pragmatic approach and made the distinction on the basis of a visual inspection as to how well the different parts can be separated. If the X-ray halos are hardly connected in the RASS images, given the short exposure, we treated them as separate units, while different maxima still engulfed by a common X-ray halo where treated as multiple-maxima clusters. This is a subjective criterion because the distinction is exposure and distance dependent. Also due to the short exposure in the RASS only a smaller fraction of the objects in this category will be found having enough source counts. Therefore a catalogue with any kind of completeness is extremely difficult to produce. A more viable approach is possibly a 
statistical characterization of the type used by Schuecker et al. (2001b) to describe the substructure frequency in the brighter REFLEX clusters. Therefore the present compilation is merely pointing out the most obvious examples of the two kinds, which could also be interesting targets for follow-up studies.

We distinguish between two classes of objects in the catalogue: close cluster pairs and clusters with two pronounced $\mathrm{X}$-ray maxima in the surface brightness distribution which were treated as single units. In total we find 10 close cluster pairs (including one triplet and one quadruplet) at closely concordant redshifts, listed in Table 10. We have not included pairs seen only in projection where the redshift of the two components is clearly different. Table 11 lists those 14 clusters which feature two or several distinct X-ray maxima.

Another complication arises in the cluster redshift determination as quite frequently several redshift groupings are found in the line-of-sight towards the cluster, indicating that several clusters and groups of galaxies are seen in projection, or narrow features of the large-scale structure (like walls and filaments) are threaded by the line-of-sight. In this case we have assigned the redshift to the cluster, which is derived from the largest number galaxies (including the bright central galaxy). In most cases the assignment is quite obvious, as illustrated by Table 12 for the combined data of our follow-up observations and the literature search. The table is ordered such that the first component refers to the chosen cluster identification while the remaining components are ordered by their redshift. The table also gives a helpful reference for those cases were discrepancies may be found in future observations. Most of the detailed information listed in the table comes from the ESO key program of the ENACS survey by Katgert et al. (1996) and Mazure et al. (1996). For each line-of-sight component we list the redshift and the number of known coincident galaxies.

\section{Comparison to other catalogues}

We have inspected the previously published catalogues of clusters detected in the RASS, to further check the completeness of our sample. Of these previous surveys the RASSB1 (De Grandi et al. 1999), the XBACS (Ebeling et al. 1996), the SGP Survey (Cruddace et al. 2002, 2003), and HIFLUGCS (Reiprich \& Böhringer 2002) surveys have made use of the material that was compiled during the ongoing REFLEX survey. Thus these samples are not independent. But since we have used a very strict automated selection criterion for the primary selection of the cluster candidates and have not included arbitrarily all we know about clusters in the REFLEX region, this is a very important completeness test and a test of the galaxy overdensity detection method applied to the COSMOS data base as described in Paper I.

The XBACS catalogue lists 5 clusters that are not included in REFLEX after four clusters are removed from the correlation (A3186, A3216, A3230, A3389), because they are located in the Magellanic cloud regions that have been excised in the present sample. For these 5 objects we have a definite reason for the exclusion. A3041: the REFLEX flux is $2.58 \times$ $10^{-12} \mathrm{erg} \mathrm{s}^{-1} \mathrm{~cm}^{-2}$ and below the current flux limit; A467: is characterized by a point source which is by about $5 \sigma$ too soft to
Table 10. List of cluster pairs and close groupings in the REFLEX catalogue.

\begin{tabular}{lrl}
\hline \hline Name & Redshift & Alternative name \\
\hline RXCJ0229.3-3332 & 0.0779 & \\
RXCJ0230.7-3305 & 0.0760 & A3027 \\
\hline RXCJ0542.1-2607 & 0.0380 & \\
RXCJ0545.4-2556 & 0.0424 & A548W \\
RXCJ0548.6-2527 & 0.0410 & A548E \\
\hline RXCJ0626.3-5341 & 0.0531 & 3391 \\
RXCJ0627.2-5428 & 0.0512 & 3395 \\
\hline RXCJ1254.3-2901 & 0.0553 & A3528a \\
RXCJ1254.6-2913 & 0.0532 & A3528b \\
\hline RXCJ1255.5-3019 & 0.0544 & A3530 \\
RXCJ1257.2-3022 & 0.0555 & A3532 \\
\hline RXCJ1327.9-3130 & 0.0482 & A3558, Shapley center \\
RXCJ1329.7-3136 & 0.0495 & Shapley center \\
RXCJ1331.5-3148 & 0.0429 & Shapley center \\
RXCJ1333.6-3139 & 0.0502 & A3562, Shapley center \\
\hline RXCJ1347.2-3025 & 0.0141 & A3574W, substructure \\
RXCJ1349.3-3018 & 0.0141 & A3574E, main cluster \\
\hline RXCJ2014.2-8038 & 0.1373 & A3664 \\
RXCJ2016.2-8047 & 0.1309 & A3666 \\
\hline RXCJ2145.9-1006 & 0.0808 & A2377 \\
RXCJ2147.0-1019 & 0.0780 & \\
\hline RXCJ2152.2-1942 & 0.0963 & A2384b \\
RXCJ2152.4-1933 & 0.0943 & A2384a \\
\hline
\end{tabular}

Table 11. List of REFLEX clusters with two or more clearly visible $\mathrm{X}$-ray maxima.

\begin{tabular}{lll}
\hline \hline Name & Morphology & Orientation \\
\hline RXCJ0034.6-0208 & two maxima & East-West \\
RXCJ0152.7-0100 & two maxima & East-West \\
RXCJ0157.4-0550 & two maxima & NE-SW \\
RXCJ0330.0-5235 & two maxima & NE-SW \\
RXCJ0624.6-3720 & two maxima & East-West \\
RXCJ0948.6-8327 & two maxima & East-West \\
RXCJ0956.4-1004 & three maxima & NE-W-S (diffuse) \\
RXCJ1305.9-3739 & two maxima & NE-SW \\
RXCJ1330.8-0512 & large elongation & towards NE \\
RXCJ2106.0-3846 & two maxima & NE-SW \\
RXCJ2157.4-0747 & two maxima & East-West \\
RXCJ2202.0-0949 & two maxima & NE-SW \\
RXCJ2218.2-0350 & two maxima & NEE-SWW \\
RXCJ2319.2-6750 & two maxima & North-South \\
\hline
\end{tabular}

be consistent with cluster emission; A3662: the REFLEX flux is $1.67 \times 10^{-12} \mathrm{erg} \mathrm{s}^{-1} \mathrm{~cm}^{-2}$, below the flux limit of REFLEX, but there is a $\sim 40 \%$ uncertainty in the flux due a low exposure of only 122 s in RASS II; A3701: (RXCJ2040.0-7114) was removed from our cluster list as explained in the Appendix; 
Table 12. Multiple redshift clustering in the line-of-sight of REFLEX clusters.

\begin{tabular}{|c|c|c|c|c|c|c|c|c|}
\hline \multirow[t]{2}{*}{ Name } & \multirow[t]{2}{*}{ Alt. name } & \multirow[b]{2}{*}{ Comp. 1} & \multicolumn{4}{|c|}{ Redshifts } & & \multirow[t]{2}{*}{ References } \\
\hline & & & Comp. 2 & Comp. 3 & Comp. 4 & Comp. 5 & Comp. 6 & \\
\hline RXCJ0003.2-3555 & A2717 & $0.0490(40)$ & $0.0720(5)$ & & & & & 2 \\
\hline RXCJ0011.3-2851 & A2734 & $0.0620(83)$ & $0.0260(5)$ & $0.1190(4)$ & $0.1410(6)$ & & & 2 \\
\hline RXCJ0013.6-1930 & A0013 & $0.0940(37)$ & $0.0270(4)$ & & & & & $\mathrm{S}, 2,140,141$ \\
\hline RXCJ0017.5-3509 & A2755 & $0.0968(23)$ & $0.1210(10)$ & & & & & $\mathrm{E}, 2,3,33,140,141$ \\
\hline RXCJ0041.8-0918 & A0085 & $0.0555(308)$ & 0.0762 & & & & & $130,148,149$ \\
\hline RXCJ0042.1-2832 & A2811 & $0.1082(29)$ & $0.0540(6)$ & & & & & $\mathrm{E}, 33,96$ \\
\hline RXCJ0056.3-0112 & A0119 & $0.0442(104)$ & $0.1400(4)$ & & & & & 2 \\
\hline RXCJ0108.8-1524 & A0151A & $0.0533(63)$ & $0.0410(25)$ & $0.1000(35)$ & & & & 1,2 \\
\hline RXCJ0110.0-4555 & A2877 & $0.0238(58)$ & $0.2470(97)$ & & & & & $12,130,131,146$ \\
\hline RXCJ0115.2+0019 & A0168 & $0.0450(76)$ & $0.0176(4)$ & $0.0720(4)$ & $0.0890(7)$ & & & $\mathrm{S}, 2$ \\
\hline RXCJ0137.2-0912 & & $0.0409(5)$ & 0.0700 & & & & & $\mathrm{~S}, 120$ \\
\hline RXCJ0152.7+0100 & A0267 & $0.2300(1)$ & $0.0592(8)$ & & & & & $55,147,159$ \\
\hline RXCJ0152.9-1345 & NGC0720 & $0.0050(3)$ & $0.8348(6)$ & & & & & $12,160,161$ \\
\hline RXCJ0157.4-0550 & A0281 & $0.1289(4)$ & 0.088 & & & & & $\mathrm{E}, 1$ \\
\hline RXCJ0202.3-0107 & A0295 & $0.0427(47)$ & $0.1020(5)$ & & & & & $2,148,162$ \\
\hline RXCJ0231.9+0114 & $\mathrm{RCS} 145^{\mathrm{d}}$ & $0.0221(10)$ & 0.2881 & & & & & $55,64,110$ \\
\hline RXCJ0337.0-3949 & A3142 & $0.1030(21)$ & $0.0660(12)$ & & & & & 2 \\
\hline RXCJ0338.4-3526 & FORNAX & $0.0051(32)$ & $0.1124(48)$ & & & & & $166,167,168,170,171$ \\
\hline RXCJ0342.8-5338 & A3158 & $0.0590(105)$ & $0.0740(4)$ & $0.1020(4)$ & & & & 2 \\
\hline RXCJ0408.2-3053 & A 3223 & $0.0600(81)$ & $0.1100(8)$ & $0.1370(8)$ & & & & $\mathrm{E}, 2$ \\
\hline RXCJ0448.2-2028 & A0514 & $0.0720(90)$ & $0.0850(4)$ & $0.1100(8)$ & & & & 2 \\
\hline RXCJ0525.5-3135 & A3341 & $0.0380(64)$ & $0.0780(15)$ & $0.1150(18)$ & $0.1310(7)$ & $0.1540(5)$ & & 2 \\
\hline RXCJ0525.8-4715 & A3343 & $0.1913(5)$ & $0.1626(6)$ & & & & & $\mathrm{E}$ \\
\hline RXCJ0530.6-2226 & A0543 & $0.1706(11)$ & $0.0850(10)$ & & & & & $\mathrm{E}, 1$ \\
\hline RXCJ0542.1-2607 & CID 36 & $0.0390(4)$ & $0.0292(2)$ & $0.0429(69)$ & & & & $\mathrm{E}, 120$ \\
\hline RXCJ0548.6-2527 & A0548E & $0.0420(237)$ & $0.0310(4)$ & $0.0630(9)$ & $0.0870(14)$ & $0.1010(21)$ & $0.1380(4)$ & 2,3 \\
\hline RXCJ0637.3-4828 & A3399 & $0.2026(11)$ & $0.1180(3)$ & $0.3796(2)$ & & & & $\mathrm{E}$ \\
\hline RXCJ0645.4-5413 & A3404 & $0.1644(2)$ & $0.3377(2)$ & & & & & $\mathrm{E}$ \\
\hline RXCJ0658.5-5556 & $1 \mathrm{ES} 0657^{\mathrm{h}}$ & $0.2965(78)$ & $0.0790(3)$ & & & & & E, 73,79 \\
\hline RXCJ0944.1-2116 & & $0.0077(1)$ & $0.0152(1)$ & & & & & E, 12,84 \\
\hline RXCJ1039.7-0841 & A1069 & $0.0650(35)$ & $0.1140(4)$ & & & & & 2 \\
\hline RXCJ1050.4-1250 & USGC S152 & $0.0155(6)$ & $0.0760(5)$ & & & & & $\mathrm{E}, 20,31$ \\
\hline RXCJ1141.4-1216 & A1348 & $0.1195(6)$ & 0.1392 & & & & & $\mathrm{H}, 16$ \\
\hline RXCJ1254.3-2901 & A3528(A) & $0.0542(69)$ & $0.0730(9)$ & & & & & 2 \\
\hline RXCJ1327.9-3130 & A3558 & $0.0480(341)$ & $0.0320(4)$ & $0.0771(6)$ & $0.1285(7)$ & & & $1,14,44,102,141,148$ \\
\hline RXCJ1329.7-3136 & A3558(B) & $0.0488(57)$ & $0.1823(4)$ & & & & & $3,14,44,63,101,102$ \\
\hline RXCJ1330.8-0152 & A1750 & $0.0852(46)$ & $0.1492(3)$ & & & & & $1,41,96$ \\
\hline RXCJ1333.6-3139 & A3562 & $0.0490(114)$ & $0.0367(4)$ & & & & & 1,141 \\
\hline RXCJ1347.5-1144 & & $0.4516(2)$ & $0.2090(3)$ & & & & & $\mathrm{E}$ \\
\hline RXCJ1401.6-1107 & A1837 & $0.0698(38)$ & $0.0372(14)$ & & & & & 1 \\
\hline RXCJ1952.2-5503 & A 3651 & $0.0600(79)$ & $0.1010(5)$ & & & & & 2 \\
\hline RXCJ2012.5-5649 & A3667 & $0.0556(162)$ & $0.0350(2)$ & $0.0990(5)$ & & & & $2,3,138,140,141$ \\
\hline RXCJ2032.1-5627 & A 3685 & $0.1380(5)$ & $0.2852(6)$ & & & & & $\mathrm{E}$ \\
\hline RXCJ2034.3-3429 & A3693 & $0.1240(6)$ & $0.0910(16)$ & & & & & $\mathrm{E}, 3$ \\
\hline RXCJ2034.7-3548 & A3695 & $0.0894(81)$ & $0.0934(18)$ & $0.1310(7)$ & & & & $2,140,141$ \\
\hline RXCJ2101.8-2802 & A 3733 & $0.0382(91)$ & $0.0739(4)$ & & & & & $13,14,146$ \\
\hline RXCJ2104.3-4120 & A3739 & $0.1651(2)$ & $0.0820(16)$ & & & & & $\mathrm{E}$ \\
\hline RXCJ2107.2-2526 & A 3744 & $0.0381(71)$ & $0.0650(5)$ & & & & & 2 \\
\hline RXCJ2146.3-5717 & A3806 & $0.0760(99)$ & $0.0540(9)$ & $0.1380(4)$ & & & & 2 \\
\hline RXCJ2146.9-4354 & A3809 & $0.0620(94)$ & $0.0910(4)$ & $0.1100(10)$ & $0.1410(11)$ & $0.1520(4)$ & & 2 \\
\hline RXCJ2154.1-5751 & A3822 & $0.0760(84)$ & $0.0390(4)$ & $0.0520(4)$ & $0.1020(4)$ & & & 2 \\
\hline RXCJ2158.3-2006 & A2401 & $0.0570(23)$ & $0.0884(2)$ & $0.0930(5)$ & & & & 2,3 \\
\hline RXCJ2158.4-6023 & A 3825 & $0.0750(61)$ & $0.1040(17)$ & $0.1190(4)$ & & & & 2,3 \\
\hline RXCJ2234.5-3744 & A3888 & $0.1510(70)$ & $0.2077(7)$ & & & & & 1,44 \\
\hline RXCJ2249.9-6425 & A3921 & $0.0940(32)$ & $0.1340(4)$ & & & & & $\mathrm{E}, 3$ \\
\hline RXCJ2312.3-2130 & A2554 & $0.1108(35)$ & $0.0707(5)$ & & & & & 38,127 \\
\hline RXCJ2321.5-4153 & A3998 & $0.0894(16)$ & $0.0665(3)$ & & & & & $\mathrm{S}, 16,31,33,48$ \\
\hline RXCJ2336.2-3136 & S1136 & $0.0643(2)$ & $0.0260(3)$ & & & & & $15,52,26$ \\
\hline RXCJ2354.2-1024 & A2670 & $0.0765(219)$ & $0.1506(13)$ & & & & & $66,86,105$ \\
\hline
\end{tabular}

The footnotes in the alternative name Col. (2) are explained in Table 6. 
A3716: has no exposure in RASS II (This is implicitly accounted for in the construction of the sky coverage map). Thus no cluster has been missed in REFLEX which is included in XBACS. There are further some nomenclature problems that are encountered in this comparison: A189 in XBCAS is a misclassification since the Abell cluster is about half a degree away and the correct identification should be NGC 533 as in REFLEX, A1664 is identical with A3541, A3017 in XBACS is identified with A3016 in REFLEX. Three clusters are listed with two separate components in XBACS which are treated as one source in the present compilation: A901, A1631, and A 1750 .

A comparison with the SGP sample by Cruddace et al. $(2002,2003)$ which is based on the same X-ray source catalogue and source characterization as REFLEX but used a different optical search method, shows only two additional clusters with a recently determined flux above the REFLEX flux limit: RXCJ2356.0-0129 which is here treated as a double source where each component falls below the flux limit, and RXCJ0016.3-3121 which was for the REFLEX catalogue deblended from a soft contaminating component and therefore fell below the flux limit after this treatment. Six clusters listed in the SGP sample catalogue with a flux above the REFLEX flux limit have slightly lower fluxes in REFLEX and are scattered below the REFLEX flux limit: RXCJ2306.8-1324, RXCJ0048.6-2114, RXCJ0108.5-4021, RXCJ0212.8-4707，RXCJ0244.1-2611，RXCJ0248.2-0216. They will be included in REFLEX II. The following 9 clusters in the SGP region are only listed in the REFLEX catalogue: RXCJ0034.6-0208, RXCJ0043.4-2037, RXCJ0132.6-0804, RXCJ0250.2-2129, RXCJ0301.6+0155, RXCJ2211.7-0350, RXCJ2248.5-1606, RXCJ2251.7-3206, RXCJ2306.6-1319. Note that already four of these nine objects have a problematic identification, as discussed in the Appendix. Therefore the missing of these clusters is to a large part not a real incompleteness but a classification problem.

The southern RASS Bright sample, compiled from an earlier version of the ESO key program X-ray cluster identification list based on the RASS I data set (De Grandi et al. 1999), contains two additional clusters that should be included in REFLEX. One was again found in our supplementary search for clusters among the sources found to be extended by the GCA analysis, RXCJ2129.6+0005 at $z=0.2347$. This source and RXJ0600.5-4846 were not flagged by the optical correlation. Since these objects were not automatically included through the correlation with the COSMOS data we have not included them in the present list. RXCJ0528.9-3927 listed by De Grandi et al. is removed from the present list as explained in the Appendix. Two further objects from this catalogue have been removed from our sample in earlier steps of the identification RXJ2136.4-6224 is a point-like X-ray source coincident with a Seyfert 1 galaxy at $z=0.0588$ and RXJ2253.9-5812 is a point source with a soft spectrum coincident with the radio source PMNJ2253-5812. All the objects listed in the HIFLUGCS sample by Reiprich \& Böhringer (2002) in the REFLEX survey area are included in the present catalogue.

\section{Discussion and conclusions}

More than 100000 X-ray sources were identified in the ROSAT All-Sky Survey (Voges et al. 1999) and more than $10 \%$ of the $\mathrm{X}$-ray sources in the sky away from the Galactic plane are expected to be galaxy clusters for the relevant range of flux limits. Thus taking into account the surely lower detection efficiency for extended cluster X-ray sources and the Galactic source population one can easily expect about 6000-8000 clusters among the X-ray sources in the bright and faint ROSAT All-Sky Survey catalogue. The REFLEX cluster sample with a flux limit of $3 \times 10^{-12} \mathrm{erg} \mathrm{s}^{-1} \mathrm{~cm}^{-2}$ is therefore only the tip of the iceberg of the RASS cluster population. However, the REFLEX sample is constructed from a selection with a relatively high flux limit, in order to yield a sample of high quality. The median number of detected source photons for the REFLEX clusters is 79 photons. Relatively safe source detections, e.g. as listed in the RASS faint source catalogue, can still be obtained from a detection of only 6 photons in a detection aperture with 2 arcmin radius, which for the typical exposure of about $400 \mathrm{~s}$ and a typical background in the hard band (channel 52-201) of about $3 \times 10^{-4} \mathrm{cts} \mathrm{s}^{-1} \operatorname{arcmin}^{-2}$ would contain about 1.5 background photons. Thus a detection of 6 photons corresponds roughly to a $3.7 \sigma$ background enhancement and roughly to a flux limit of $3 \times 10^{-13} \mathrm{erg} \mathrm{s}^{-1} \mathrm{~cm}^{-2}-$ a flux limit one order of magnitude below the REFLEX cut. Thus the RASS provides the prospect of finding many more clusters than presented here, but the price is a much lower quality of the X-ray characteristics as well as a much more difficult job for the definitive source identification ${ }^{1}$.

With the relatively high flux cut the high quality of the REFLEX sample is characterized by the following most important properties: (i) a flux determination with a typical accuracy of 10-20\%; (ii) a large fraction of the cluster X-ray sources can be characterized as extended $(\sim 80 \%)$ which adds very much to a safe identification; (iii) we can in general obtain a meaningful spectral hardness ratio which allows further discrimination in our identification; (iv) we arrive at a selection function which is almost homogeneous across the sky. At an even higher flux limit, e.g. $6 \times 10^{-12} \mathrm{erg} \mathrm{s}^{-1} \mathrm{~cm}^{-2}$, only 9 clusters are not flagged as extended $\mathrm{X}$-ray sources by our analysis. The spatially very homogeneous selection function ensures for example that, for a detection limit of 10 photons used in most of our spatial distribution analysis, the flux limit is reached in $97 \%$ of the sky and only small corrections apply for the remaining region. The selection function is also characterized by the analysis of the source photon count distribution shown in Fig. 25 of Paper I, from which we conclude that only about 14 clusters might be missed if the correction for the lower sensitivity in some regions of the REFLEX survey is neglected. Therefore the cosmological results derived by us in the series of REFLEX papers could also be reproduced in good approximation if the angular

\footnotetext{
1 Already the number of spurious sources is expected to be of the order of 1000 in the REFLEX area for the mentioned low flux limit. This is in comparison to a few Thousand clusters to be found in the same area not unreasonable. But good extra information as for example optical or other wavelength data is mandatory for an identification in this case.
} 
modulation of the selection function given in Tables 8 and 9 were neglected.

Since the completion of the optical follow-up observations for REFLEX we have already embarked on the extension of this survey, REFLEX II which is now close to complete down to a flux limit of $1.8 \times 10^{-12} \mathrm{erg} \mathrm{s}^{-1} \mathrm{~cm}^{-2}$ including more than 800 clusters. We stress that in addition to the fact that the $\mathrm{X}$-ray parameters become less accurate at these lower fluxes, the identification work becomes significantly harder: the optical counter parts are less striking on for example the optical DSS images, it is more difficult to detect and catch the signature of contaminating AGN, and the spatial modulation of the selection function can no longer be neglected. Therefore the gain in the statistics by the increase of the sample size has a price and there is some optimum quality and size of the cluster sample, depending of course on the application, for which the flux limit should not be much lower than that of REFLEX.

Further work on the identification of RASS clusters is useful if the RASS data can be combined with survey information from other wavelengths. A good example is the Sloan Digital Sky Survey. Based on the parent SDSS cluster sample from Sheldon et al. (2001) we have demonstrated that we can almost recover the REFLEX X-ray luminosity function for clusters down to a flux limit in the RASS of $8 \times 10^{-13} \mathrm{erg} \mathrm{s}^{-1} \mathrm{~cm}^{-2}$. Work in progress on the combined detection of galaxy clusters in X-rays (RASS) and the optical (SDSS) extends these detections to even lower fluxes (Schuecker et al. 2004) recovering an areal density of about 0.5 clusters $\mathrm{deg}^{-2}$.

The high quality of the REFLEX sample makes the present catalogue a useful basis for the careful selection of targets for detailed follow-up studies at all wavelengths. A deep XMM-Newton follow-up study has been targeted at a complete sample of $13 \mathrm{X}$-ray luminous REFLEX clusters $\left(L_{\mathrm{x}} \geq 1 \times\right.$ $10^{45} \mathrm{erg} \mathrm{s}^{-1}$ ) in the redshift interval 0.27 to 0.31 (Zhang et al. 2003) to study for example the evolution of the cluster population at the high mass end, from $z \sim 0.3$ to the present. One first result of this study is that all 13 clusters of the sample show X-ray emission which is dominated by thermal, diffuse intracluster medium radiation and only two clusters have a point source contribution of at most $30 \%$. This provides a very nice confirmation for the REFLEX cluster sample, since at this upper end of the redshift distribution of the REFLEX clusters it is already quite difficult to recognize the point source contamination of the cluster emission. The same clusters form also the subject of a very detailed optical study with the VLT in the frame of a Large Program to study among other objectives the galaxy dynamics, the characteristics of the galaxy population, and the supernova rate. Recently a Large XMM-Newton follow-up program has been approved, to study in detail 33 clusters from REFLEX selected in such a way that they form a volume complete sample (with a complex but known volume structure), that they cover the X-ray luminosity range almost homogeneously, and that they are optimally observed with the field-of-view of the XMM-Newton instruments. The main goal of this survey is to understand the statistics of cluster structure and the scaling relations of observable and physical parameters, like the very important $\mathrm{X}$-ray luminosity-mass and temperature-mass relations. These relations form the backbone of the cosmological application of cluster samples like REFLEX.

Further applications of the REFLEX catalogue are in progress involving the study of radio haloes, Sunyaev-Zeldovich observations, correlations with microwave background data (e.g., WMAP), and gravitational lensing studies. The cosmological applications of the REFLEX sample demonstrates the power of using clusters of galaxies as cosmological probes in a way complementary to studies based on supernovae and on the microwave background. Therefore we hope that in a next step a new X-ray all-sky survey which goes much deeper, covers a larger X-ray band width, and provides a better angular resolution will enable us with much higher precision to test cosmological models and determine cosmological parameters. There is a constant effort in the X-ray community to make such a project and such an important progress possible.

Note added in proof. One of the objects in the catalogue, RXCJ1350.7-3343, is found in a recently obtained XMM observation to show X-ray emission clearly dominated by a central AGN.

Acknowledgements. We like to thank the ROSAT team at MPE for the support with the data reduction of the ROSAT All-Sky Survey and the staff of ESO La Silla for the technical support during the numerous observing runs for the ESO key programme. We also thank G. Vettolani, W. C. Seitter, K. A. Romer, U. G. Briel, H. Ebeling, R. Dümmler, T. H. Reiprich, R. A. Schwarz, S. Molendi, H. Gursky, and D. Yentis for help during the programme. We also thank the referee for very useful comments.

The production of the COSMOS digital optical data base, based on scans of the UK Schmidt southern sky survey IIIa-J plates, and the development of techniques for detecting cluster candidates in the COSMOS data, was a major project, requiring the coordinated efforts of Harvey MacGillivray (ROE), Daryl Yentis, Brad Stuart (NRL), and John Wallin (George Mason University).

This research also made use of the NASA/IPAC Extragalactic Database (NED), which is operated by the Jet Propulsion Laboratory, California Institute of Technology, under contract with NASA. P.S. acknowledges the support by the Verbundforschung under grant No. 50 OR 93065.

\section{References}

Abell, G. O. 1958, ApJS, 3, 211

Abell, G. O., Corwin, H. G., \& Olowin, R. P. 1989, ApJS, 70, 1

Allen, S. W., Edge, A. C., Fabian, A. C., et al. 1992, MNRAS, 259, 67

Alonso, M. V., Valotto, C., Lambas, D. G., \& Muriel, H. 1999, MNRAS, 308, 618

Andernach, H., \& Tago, E. 1998, in Proc. of the 12th Potsdam Cosmology Workshop: Large Scale Structure: Tracks and Traces, ed. V. Mueller, S. Gottloeber, J. P. Muecket, \& J. Wambsganss (World Scientific), 147

Andernach, H. 2002, private communication, upgrade of the compilation by Andernach \& Tago (1998)

Anders, E., \& Grevesse, N. 1989, Geochim. Cosmochim. Acta, 53, 197

Arnaud, M., Hughes, J. P., Forman, W., et al. 1992, ApJ, 390, 345

Bade, N., Fink, H. H., Engels, D., et al. 1995, A\&A, 110, 469

Bardelli, S., Zucca, E., Vettolani, G., et al. 1994, MNRAS, 267, 665 
Bardelli, S., Pisani, A., Ramella, M., Zucca, E., \& Zamorani, G. 1998, MNRAS, 300, 589

Bardelli, S., Zucca, E., \& Baldi, A. 2001, MNRAS, 320, 387

Barrena, R., Biviano, A., Ramella, M., Falco, E. E., \& Seitz, S. 2002, A\&A, 386, 816

Barton, E., Geller, M. J., Ramella, M., Marzke, R. O., \& Da Costa, L. N. 1996, AJ, 112, 871

Batuski, D. J., Burns, J. O., Newberry, M. V., et al. 1991, AJ, 101, 1983

Batuski, D. J., Miller, C. J., Slinglend, K. A., et al. 1999, ApJ, 520, 491

Bauer, F. E., Condon, J. J., Thuan, T. X., \& Broderick, J. J. 2000, ApJS, 129, 547

Beers, T. C., Forman, W., Huchra, J. P., Jones, C., \& Gebhardt, K. 1991, AJ, 102, 1581

Beers, T. C., Gebhardt, K., Huchra, J. P., Forman, C., \& Bothun, G. D. 1992, ApJ, 400, 410

Beers, T. C., Kriessler, J. R., Bird, C. M., \& Huchra, J. P. 1995, 109, 874

Bernardi, M., Alonso, M. V., da Costa, L. N., et al. 2002, AJ, 123, 2990

Blakeslee, J. P., \& Tonry, J. 1992, AJ, 103, 1457

Böhringer, H., Guzzo, L., Collins, C. A., et al. 1998, The Messenger, 94, 21

Böhringer, H., Voges, W., Huchra, J. P., et al. 2000, ApJS, 129, 435

Böhringer, H., Schuecker, P., Guzzo, L., et al. 2001a, A\&A, 369, 826 (Paper I)

Böhringer, H., Schuecker, P., Lynam, P., et al. 2001b, The Messenger, 106, 24

Böhringer, H., Schuecker, P., Komossa, S., et al. 2001c, in Mapping the Hidden Universe, Proc. of a workshop in Guanajuato, Mexico, Fabruary 2000, ed. R. C. Kraan-Korteweg, P. A. Henning, \& H. Andernach, 93 [arXiv: astro-ph/0011461]

Böhringer, H., Collins, C. A., Guzzo, L., et al. 2002, ApJ, 566, 93 (Paper IV)

Burns, J. O., Ledlow, M. J., Loken, C., et al. 1996, ApJ, 467, L49

Caccianiga, A., Maccacaro, T., Wolter, A., Della Ceca, R., \& Gioia, I. M. 2000, A\&AS, 144, 247

Caldwell, N., \& Rose, J. A. 1997, AJ, 113, 492

Caretta, C. A., Maia, M. A. G., Kawasaki, W., \& Willmer, C. N. A. 2002, AJ, 123, 1200

Carter, D., \& Malin, D. F. 1983, MNRAS, 203p, 49

Cavaliere, A., \& Fusco-Femiano, R. 1976, A\&A, 49, 137

Chen, J., Huchra, J. P., McNamara, B., \& Mader, J. 1998, BAAS, 30, 1307 - data located at http://cfa-www.harvard.edu/ huchra/clusters

Christiani, S., de Souza, R., D’Odorico, S., Lund, G., \& Quintana, H. 1987, A\&A, 179, 108

Collins, C. A., Guzzo, L., Nichol, R. C., \& Lumsden, S. L. 1995, MNRAS, 274, 1071

Collins, C. A., Guzzo, L., Böhringer, H., et al. 2000, MNRAS, 319, 939, (Paper II)

Colless, M., Dalton, G., \& Maddox, S. 2001, MNRAS, 328, 1039

Collins, M., \& Hewett, P. 1987, MNRAS, 224, 453

Condon, J. J., Cotton, W. D., Greisen, E. W., et al. 1998, AJ, 115, 1693

Couch, W. J., \& Sharples, R. M. 1987, MNRAS, 229, 423

Couch, W. J., Barger, A. J., Smail, I., Ellis, R. S., \& Sharples, R. M. 1998, ApJ, 497, 188

Crawford, C. S., Edge, A. C., Fabian, A. C., et al. 1993, 274, 75

Crawford, C. S., Edge, A. C., Fabian, A. C., et al. 1995, MNRAS, 274, 75

Crawford, C. S., Allen, S. W., Ebeling, H., Edge, A. C., \& Fabian, A. C. 1999 , MNRAS, 306, 857
Cruddace, R., Voges, W., Böhringer, H., et al. 2002, ApJS, 140, 239

Cruddace, R., Voges, W., Böhringer, H., et al. 2003, ApJS, 144, 299

da Costa, L. N., Willmer, C., Pellegrini, P. S., \& Chincarini, G. 1987, AJ, 93, 1338

da Costa, L. N., Pellegrini, P. S., Davis, M., et al. 1991, ApJS, 75, 935

da Costa, L. N., Willmer, C. N. A., Pellegrini, P. S., et al. 1998, AJ, 116,1

Dale, D. A., Giovanelli, R., Haynes, M. P., \& Scodeggio, M. 1998, AJ, 115,418

Dalton, G. B., Efstathiou, G., Maddox, S. J., \& Sutherland, W. J. 1994, MNRAS, 269, 151

Dalton, G. B., Maddox, S. J., Sutherland, W. J., \& Efstathiou, G. 1997, MNRAS, 289, 263

Dantas, C. C., De Carvalho, R. R., Capelato, H. V., \& Mazure, A. 1997, ApJ, 485, 447

Davoust, E., \& Considere, S. 1995, A\&AS, 110, 19

De Carvalho, R. R., Ribiero, A. L. B., Capelato, H. V., \& Zepf, S. E. 1997, ApJS, 110, 1

De Grandi, S., Molendi, S., Böhringer, H., \& Voges, W. 1997, ApJ, 486, 738

De Grandi, S., Böhringer, H., Guzzo, L., et al. 1999, ApJ, 514, 148

den Hartog, R. 1995, Ph.D. Thesis, Univ. Leiden

den Hartog, R., \& Katgert, P. 1996, MNRAS, 279, 349

de Vaucouleurs, G., de Vaucouleurs, A., Corwin, H. G., Jr., et al. 1991, Third Reference Catalogue of Bright Galaxies, Vol. 1-3 (Berlin, Heidelberg, New York: Springer Verlag)

Di Nella, H., Couch, W. J., Paturel, G., \& Parker, Q. A. 1996, MNRAS, 283, 367

Dickey, J. M., \& Lockman, F. J. 1990, ARA\&A, 28, 215

Dressler, A., \& Shectman, S. A. 1988, AJ, 95, 284

Dressler, A., Schechter, P. L., \& Rose, J. A. 1986, AJ, 91, 1058

Drinkwater, M. J., Proust, D., Parker, Q. A., Quintana, H., \& Slezak, E. 1999, PASA, 16, 113

Drinkwater, M. J., Gregg, M. D., Holman, B. A., \& Brown, M. J. I. 2001, MNRAS, 326, 1076

Durret, F., Felenbork, P., Lobo, C., \& Slezak, E. 1998, A\&AS, 129, 28

Ebeling, H., Voges, W., Böhringer, H., \& Edge, A. C. 1993, A\&A, 275,360

Ebeling, H., \& Maddox, S. J. 1995, MNRAS, 275, 1155

Ebeling, H., Voges, W., Böhringer, H., et al. 1996, MNRAS, 281, 799

Ebeling, H., Edge, A. C., Böhringer, H., et al. 1998, MNRAS, 301, 881

Ebeling, H., Edge, A. C., Allen, S. W., et al. 2000a, MNRAS, 318, 333

Ebeling, H., Jones, L. R., Perlman, E., et al. 2000b, ApJ, 534, 133

Ebeling, H., Edge, A. C., \& Henry, J. P. 2001, ApJ, 553, 668

Ebeling, H., Mullis, C. R., \& Tully, R. B. 2002, ApJ, 580, 774

Edge, A. C., \& Stewart, G. C. 1991, MNRAS, 252, 414

Edge, A. C., Steward, G. C., \& Fabian, A. C. 1992, MNRAS, 258, 177

Einasto, M., Einasto, J., Tago, E., Mueller, V., \& Andernach, H. 2001, AJ, 122, 2222

Ellis, R. S., Gray, P. M., Carter, D., \& Godwin, J. 1984, MNRAS, 206, 285

Ettori, S., Guzzo, L., \& Tarenghi, M. 1995, MNRAS, 276, 689

Fadda, D., Girardi, M., Giuricin, G., Mardirossian, F., \& Mezzetti, M. 1996, ApJ, 473, 670

Fairall, A. A. 1984, AJ, 210, 69

Fairall, A. P., Willmer, C. N. A., Calderon, J. H., et al. 1992, AJ, 103, 11

Falco, E. E., Kurtz, M. J., Geller, M. J., et al. 1999, PASP, 111, 438

Fetisova, T. S., Kuznetsov, D. Yu., Lipovetskii, V., Starobinsky, A. A., \& Olowin, R. P. 1993, Astron. Lett., 19, 198

Fischer, J.-U., Hasinger, G., Schwope, A. D., et al. 1998, AN, 319, 347 
Garilli, B., Maccagni, D., \& Vettolani, G. 1991, AJ, 101, 795

Garilli, B., Maccagni, D., \& Tarenghi, M. 1993, A\&AS, 100, 33

Gioia, I. M., Maccacaro, T., Morris, S. L., et al. 1990, ApJS, 72, 567

Gioia, I. M., Henry, J. P., Mullis, C. R., et al. 2003, ApJS, 149, 29

Goto, T., Sekiguchi, M., Nichol, R. C., et al. 2002, AJ, 123, 1807

Grazian, A., Omizzolo, A., Corbally, C., et al. 2002, AJ, 124, 2955

Green, M. R., Godwin, J. G., \& Peach, J. V. 1988, MNRAS, 234, 1051

Green, M. R., Godwin, J. G., \& Peach, J. V. 1990, MNRAS, 243, 159

Green, M. R., Godwin, J. G., \& Peach, J. V. 1999, AJ, 118, 1468

Griffith, M. R., \& Wright, A. E. 1993, AJ, 105, 1666

Guzzo, L., Böhringer, H., Schuecker, P., et al. 1999, The Messenger, 95,27

Heckman, T. M., O’Dea, C. P., Baum, S. A., \& Laurikainen, E. 1994, ApJ, 428, 65

Henry, J. P., Gioia, I. M., Huchra, J. P., et al. 1995, ApJ, 449, 422

Henry, J. P., \& Mullis, C. R. 1997 (priv. com.)

Henry, J. P., Gioia, I. M., Mullis, C. R., et al. 2001, ApJ, 553, L109

Hewitt, A., \& Burbidge, G. 1991, ApJS, 75, 297

Heydon-Dumbleton, N. H., Collins, C. A., \& MacGillivray, H. T. 1989, MNRAS, 238, 379

Hickson, P., Mendes de Oliveira, C., Huchra, J. P., \& Palumbo, G. G. 1992, ApJ, 399, 353

Hilker, M., Infante, L., Vieira, G., Kissler-Patig, M., \& Richtler, T. 1999, A\&A, 134, 75

Huchra, J. P., Postman, M., Geary, J., \& Geller, M. J. 1991, preprint (cited in Postman, Huchra \& Geller 1992)

Huchra, J., Latham, D. W., Da Costa, L. N., Pellegrini, P. S., \& Willmer, C. N. A. 1993, AJ, 105, 1637

Huchra, J. P., Vogeley, M. S., \& Geller, M. J. 1999, ApJS, 121, 287

Huchtmeier, W. K. 1994, A\&A, 286, 389

Jones, C., \& Forman, W. 1999, ApJ, 511, 65

Jorgensen, I., Franx, M., \& Kjaergaard, P. 1995, MNRAS, 276, 1341

Kaldare, R., Colless, M., Raychaudhury, S., \& Peterson, B. A. 2003, MNRAS, 339, 652

Kapahi, V. K., Athreya, R. M., van Bruegel, W., McCarthy, P. J., \& Subrahmanya, C. R. 1998, ApJS, 118, 275

Katgert, P., Mazure, A., Perea, J., et al. 1996, A\&A, 310, 8

Katgert, P., Mazure, A., den Hartog, R., et al. 1998, A\&AS, 129, 399

Kerscher, M., Mecke, K., Schuecker, P., et al. 2001, A\&A, 377, 1

Koranyi, D. M., \& Geller, M. J. 2002, AJ, 123, 100

Kristian, J., Sandage, A., \& Westphal, J. A. 1978, ApJ, 221, 383

Lauberts, A., \& Valentijn, E. A. 1989, The surface photometry catalogue of the ESO-Uppsala galaxies, ESO, Garching, Germany

Lauer, T. R., \& Postman, M. 1994, ApJ, 425, 418

Lebedev, V. S., \& Lebedeva, I. A. 1992, A Compilation of Redshifts of Clusters of Galaxies, Special Astrophys. Obs. unpublished

Ledlow, M. J., \& Owen, F. N. 1995, AJ, 110, 1959

Ledlow, M. J., Loken, C., Burns, J. O., Owen, F. N., \& Voges, W. 1999, ApJ, 516, L53

Lemonon, L., Pierre, M., Hunstead, R., et al. 1997, A\&A, 326, 34

Liang, H., Lemonon, L., Valtchanov, I., Pierre, M., \& Soucail, G. 2000, A\&A, 363, 440

Loveday, J., Peterson, B. A., Maddox, S. J., \& Efstathiou, G. 1996, ApJS, 107, 201

Lumsden, S. L., Nichol, R. C., Collins, C. A., \& Guzzo, L. 1992, MNRAS, 258, 1

Lucey, J. R., Dickens, R. J., Mitchell, R. J., \& Dawe, J. A. 1983, MNRAS, 203, 545

MacGillivray, H. T., \& Stobie, R. S. 1984, Vistas Astr., 27, 433

MacGillivray, H. T., Law, S. D., Cruddace, R. G., et al. 1994, in Cosmological Aspects of X-ray Clusters of Galaxies, ed. W. C. Seitter (Netherlands: Kluwer Academic Publ.), 339

Machalski, J., \& Condon, J. J. 1999, ApJS, 123, 41
Mahdavi, A., Böhringer, H., Geller, M. J., \& Ramella, M. 2000, ApJ, 534,114

Malamuth, E. M., Kriss, G. A., Dixon, V. D., Ferguson, H. C., \& Richie, C. 1992, AJ, 104, 495

Mamon, G. A., Parker, Q. A., \& Proust, D. 2001, PASA, 18, 232

Markevitch, M. 1998, ApJ, 504, 27

Marzke, R. O., Huchra, J. P., \& Geller, M. J. 1996, AJ, 112, 1803

Matsumoto, H., Pierre, M., Tsuru, T. G., \& Davis, D. S. 2001, A\&A, 374, 28

Matthews, L. D., \& van Driel, W. 2000, A\&AS, 143, 421

Mathewson, D. S., Ford, V. L., \& Buchhorn, M. 1992, ApJS, 81, 413

Mathewson, D. S., \& Ford, V. L. 1996, ApJS, 107, 97

Mauch, T., Murphy, T., Buttery, H. J., et al. 2003, MNRAS, 342, 1117

Maurogordato, S., Proust, D., Cappi, A., Slezak, E., \& Martin, J. M. 1997, A\&AS, 123, 411

Maurogordato, S., Proust, D., Beers, T. C., et al. 2000, A\&A, 355, 848

Mazure, A., Katgert, P., den Hartog, R., et al. 1996, A\&A, 310, 31

McCarthy, P. J., Kapahi, V. K., van Bruegel, W., et al. 1996, ApJS, 107,19

Melnick, J., \& Quintana, H. 1981, AJ, 86, 1567

Merrifield, M. R., \& Kent, S. M. 1991, AJ, 1001, 783

Metcalfe, N., Fong, R., Shanks, T., \& Kilkenny, D. 1989, MNRAS, 236, 207

Mieske, S., Hilker, M., \& Infante, L. 2002, A\&A, 383, 823

Minniti, D., Kissler-Patig, M., Goudfrooij, P., \& Meylan, G. 1998, AJ, 115,121

Mohr, J. J., Geller, M. J., \& Wegner, G. 1996, AJ, 112, 1816

Mould, J. R., Staveley-Smith, L., Schommer, R. A., et al. 1991, ApJ, 383,467

Mulchaey, J. S., Davis, D. S., Mushotzky, R. F., \& Burstein, D. 1996. ApJ, 456, 80

Müller, K., Wegner, G., Raychaudhury, S., \& Freudling, W. 1999, A\&AS, 140, 327

Muriel, H., Nicotra, M., \& Lambas, D. G. 1991, AJ, 101, 1997

Muriel, H., Nicotra, M. A., \& Lambas, D. G. 1995, AJ, 110, 1032

Oegerle, W. R., \& Hill, J. M. 2001, AJ, 122, 2858

Olowin, R., De Souza, R. E., \& Chincarini, G. 1988, A\&AS, 73, 125

Ortiz-Gil, A., Guzzo, L., Schuecker, P., Böhringer, H., \& Collins, C. A. 2003, MNRAS, 348, 325

Owen, F. N., Ledlow, M. J., \& Keel, W. C. 1995, AJ, 109, 14

Owen, F. N., Ledlow, M. J., Keel, W. C., \& Morrison, G. E. 1999, AJ, 118,633

Patten, D. R., Carlberg, R. G., Marzke, R. O., Pritchet, C. J., \& Da Costa, L. N. 2000, ApJ, 536, 153

Peacock, J. A., \& West, M. 1992, MNRAS, 259, 494

Pena, M., Ruiz, M. T., \& Maza, J. 1991, A\&A, 251, 417

Peterson, B. A., Wright, A. E., Jauncey, D. L., \& Condon, J. J. 1997, 232,400

Piccinotti, G., Mushotzky, R. F., Boldt, E. A., et al. 1982, ApJ, 253, 485

Pierre, M., Böhringer, H., Ebeling, H., et al. 1994, A\&A, 290, 725

Pierre, M., Oukbir, J., Dubreuil, D., et al. 1997, A\&AS, 124, 283

Pismis, P., Mampaso, A., Manteiga, M., Recilllas, E., \& Cruz Gonzales, G. 1995, AJ, 109, 140

Postman, M., Huchra, J. P., \& Geller, M. J. 1992, ApJ, 384, 404

Postman, M., \& Lauer, T. R. 1995, ApJ, 440, 28

Quintana, H., \& Ramirez, A. 1990, AJ, 1424

Quintana, H., \& de Souza, R. 1993, A\&AS, 101, 475

Quintana, H., \& Ramirez, A. 1994, ApJS, 96, 343

Quintana, H., Fouque, P., \& Way, M. J. 1994, A\&A, 283, 722

Quintana, H., Ramirez, A., Melnick, J., Raychaudhury, S., \& Slezak, E. 1995, AJ, 110, 463

Quintana, H., Ramirez, A., \& Way, M. J. 1996a, AJ, 111, 603 
Quintana, H., Ramirez, A., \& Way, M. J. 1996b, AJ, 112, 36

Ramella, M., Focardi, P., \& Geller, M. J. 1996, A\&A, 312, 745

Ramella, M., Geller, M. J., Pisani, A., \& da Costa, L. N. 2002, AJ, 123, 2976

Ramirez, R. C., \& de Souza, R. E. 1998, ApJ, 496, 693

Ratcliffe, A., Shanks, T., Parker, Q. A., et al. 1998, MNRAS, 300, 417

Raymond, J. C., \& Smith, B. W. 1977, ApJS, 35, 419

Reimers, D., Köhler, T., \& Wisotzki, L. 1996, A\&AS, 115, 235

Reiprich, T. H., \& Böhringer, H. 2002, ApJ, 567, 716

Retzlaff, J., Borgani, S., Gottlöber, S., Klypin, A., \& Müller, V. 1998, New Astron., 3, 631

Richter, O.-G. 1987, A\&AS, 67, 261

Rizza, E., Burns, J. O., Ledlow, M. J., et al. 1998, MNRAS, 301, 328

Romer, A. K. 1994, Ph.D. Thesis (Liverpool Univ.)

Romer, A. K., Collins, C. A., Böhringer, H., et al. 1994, Nature, 372, 75

Rose, J. A., Gaba, A. E., Christiansen, W. A., et al. 2002, AJ, 123, 1216

Schindler, S. 2000, A\&AS, 142, 433

Schuecker, P., Böhringer, H., Guzzo, L., et al. 2001a, A\&A, 368, 86 (Paper III)

Schuecker, P., Böhringer, H., Reiprich, T. H., \& Feretti, L. 2001b, A\&A, 378, 408

Schuecker, P., Guzzo, L., Collins, C. A., \& Böhringer, H. 2002, MNRAS, 335, 807 (Paper VI)

Schuecker, P., Böhringer, H., Collins, C. A., \& Guzzo, L. 2003a, A\&A, 398, 867, (Paper VII)

Schuecker, P., Caldwell, R. R., Böhringer, H., et al. 2003b, A\&A, 402, 53

Schuecker, P., Böhringer, H., \& Voges, W. 2004, A\&A, 420, 61

Schwope, A., Hasinger, G., Lehmann, I., et al. 2000, AN, 321, 1

Sharples, R. M., Ellis, R. S., \& Gray, P. M. 1998, MNRAS, 231, 479

Shectman, S. A., Landy, S. D., Oemler, A., et al. 1996, ApJ, 470, 172

Sheldon, E. S., Annis, J., Böhringer, H., et al. 2001, ApJ, 554, 881

Slinglend, K., Batuski, D., Miller, C., Michaud, K., \& Hill, J. M. 1998, ApJS, 115, 1

Smith, R. J., Lucey, J. R., Hudson, M. J., Schlegel, D. J., \& Davies, R. L. 2000, MNRAS, 313, 469

Sodre, L., Jr., Capelato, H. V., Steiner, J. E., Proust, D., \& Mazure, A. 1992, MNRAS, 259, 233

Sodre, L., Jr., Proust, D., Capelato, H. V., et al. 2001, A\&A, 377, 428

Solanes, J. M., \& Stein, P. 1998, A\&AS, 131, 221

Stark, A. A., Gammie, C. F., Wilson, R. W., et al. 1992, ApJS, 79, 77

Stein, P. 1996, A\&AS, 116, 203
Stocke, J. T., Morris, S. L., Gioia, I. M., et al. 1991, ApJS, 76, 813

Strauss, M. A., Huchra, J. P., Davis, M., et al. 1992, ApJS, 83, 29

Struble, M. F., \& Rood, H. J. 1999, ApJS, 125, 35

Teague, P. F., Carter, D., \& Gray, P. M. 1990, ApJS, 72, 715

Thompson, D. J., Djorgovski, S., Vigotti, M., \& Grueff, G. 1992, ApJS, 81, 1

Tittley, E. R., \& Henriksen, M. 2001, ApJ, 563, 673

Tonry, J. L. 1985, AJ, 90, 2431

Trager, S. C., Faber, S. M., Worthey, G., \& Gonzalez, J. J. 2000, AJ, 119,1645

Trasarti-Battistoni, R. 1998, A\&AS, 130, 341

Tritton, K. P. 1972, MNRAS, 158, 277

Trümper, J. 1992, Royal Astron. Soc. Quart. J., 33, 165

Trümper, J. 1993, Science, 260, 1769

Tucker, D. L., Oemler, A., Jr., Hashimoto, Y., et al. 2000, ApJS, 130, 237

Tucker, W., Blanco, P., Rappoport, S., et al. 1998, ApJ, 496, L5

Veron-Cetty, M.-P., \& Veron, P. 2001, A\&A, 374, 92

Vettolani, G., Cappi, A., Chincarini, G., et al. 1989, A\&AS, 79, 147

Vettolani, G., Chincarini, G., Scaramella, R., \& Zamorani, G. 1990, AJ, 99, 1709

Vettolani, G., Zucca, E., Merighi, R., et al. 1998, A\&AS, 130, 323

Voges, W., Boller, T., Dennerl, K., et al. 1996, Proc. Röntgenstrahlung from the Universe, ed. H. U. Zimmermann, J. Trümper, \& H. York, MPE Report, 263, 637

Voges, W., Aschenbach, B., Boller, T., et al. 1999, A\&A, 349, 389

Way, M. J., Flores, R. A., \& Quintana, H. 1998, ApJ, 502, 134

Wegner, G., Colless, M., Baggley, G., et al. 1996, ApJS, 106, 1

Wegner, G., Colless, M., Saglia, R. P., et al. 1999, MNRAS, 305, 259

West, R. M., \& Frandsen, S. 1981, A\&AS, 44, 329

Whiteoak, J. B. 1972, AuJPh, 25, 233

Willmer, C. N. A., Focardi, P., Chan, R., Pellegrini, P. S., \& da Costa, N. L. 1991, AJ, 101, 57

Willmer, C. N. A., Maia, M. A. G., Mendes, S. O., et al. 1999, AJ, 118, 1131

York, D. G., Adelman, J., Anderson, J. E., et al. 2000, AJ, 120, 1579

Zabludoff, A. I., Huchra, J. P., \& Geller, M. J. 1990, ApJS, 74, 1

Zabludoff, A., Geller, M., Huchra, J. P., \& Vogeley, M. S. 1993, AJ, 106, 1273

Zabludoff, A., \& Mulchaey, J. S. 1998, ApJ, 496, 39

Zhang, Y.-Y., Finoguenov, A., Böhringer, H., et al. 2003, A\&A, 413, 49

Zimmermann, H. U., Becker, W., Belloni, T., et al. 1994, EXSAS User's Guide, MPE Report, No. 257 


\section{Online Material}




\section{Appendix A: Information on individual objects}

The REFLEX data base used for the cosmology work in the REFLEX papers up to number VIII is based on the catalogue version of 1999. As we have collected more information since, we went through a further critical inspection of all the catalogue entries prior to the release of this catalogue, in addition to the revision of redshifts as described above. A major route of approach was to carefully assess the nature of those objects for which the X-ray criteria left us with a not very satisfactory means of identification. Thus we inspected especially all those $\mathrm{X}$-ray sources again which appeared point-like in the RASS. The fact that an X-ray source appears point-like at the resolution of the All-Sky Survey is not yet a criterion to reject a source. An illustrative example for this is the massive, and optically very impressive cluster RXCJ1206.2-0848 at redshift $z=0.4414$ which appears in the RASS as a point source. But we use the point-like appearance here as a flag for sources to be doubly checked. On the other hand, if an X-ray source is clearly well extended, we can be sure that most of the X-ray emission is diffuse and the contribution to the X-ray flux from AGNs or radio galaxies found at other wavelength can at most be a minor fraction. In addition to the $\mathrm{X}$-ray based reinspection we have also screened again the literature for any information that could cast doubts on the cluster identification, with a special eye on contaminating AGN. One source of further observational information that was used for some clusters are pointed observations with the ROSAT PSPC and HRI detector as well as new observations with XMM-Newton and CHANDRA.

As a result of this screening procedure we could improve our identification and discarded 7 objects from the REFLEX list as described in the subsection below. We should also point out that most of the critical cases commented below were known as problematic targets to our observing team at the time of the observation. One of the observational requirements was therefore to take a spectrum of suspicious objects in the center of the X-ray emission (often bright galaxies) to critically check for the presence of an AGN. More details about the spectra of these objects will be given in Guzzo et al. (in preparation). Thus for some of the objects where the identification is still not completely certain and the standard optical means have already been used, a final decision can only come from a high angular resolution X-ray observation or for example from optical polarimetric measurements. In total we are left with 14 cases where the identification is not definitive. Following our previous philosophy to keep the objects in our working list until we can positively rule them out as cluster identifications, we have conservatively kept them in our catalogue but we have marked them in the table. We realistically expect that less than 10 are non-cluster objects. The effect this small fraction (less than 2.3\%) has on any of the cosmological applications of REFLEX is completely negligible.

In the following we provide notes on the results of our inspection including the critical cases mentioned as well as positive confirmations. These remarks should be of help for any further identification or follow-up work that may be conducted on these REFLEX objects. The comments given for each object illustrate some aspects of the manual screening process.
RXCJ0014.3-6604, A2746 (also included in XBACS) is a typical example of a point-like X-ray source which was inspected in more detail. The X-ray spectral hardness ratio is not outside the acceptable limit for cluster emission. The source appears very compact with a core radius $2 \sigma$ upper limit of about $120 h_{50}^{-1} \mathrm{kpc}$ at a redshift of $z=0.156$. This does not rule out that the X-ray emission comes from a cluster. We have five concordant redshifts including the $\mathrm{cD}$ galaxy, with no spectrum indicating an AGN and the optical image is clearly showing a galaxy cluster. Therefore, even though we cannot establish the extent of the X-ray source, we conclude that the source is most likely a cluster.

RXCJ0015.4-2350, A14, has an X-ray emission which is very faint and diffuse: about 48 source photons spread in a clumpy distribution over a region with a radius of about 12 arcmin. The cluster position was not taken as the center of the large-scale distribution but at a local maximum. The $\mathrm{cD}$ galaxy, ESO 473-G 005 at the position $001510.6,-235257.0$ is located at this highest local maximum. This is surely a good example of a dynamically young cluster with a size slightly smaller than the Virgo cluster. Even though there is a small probability that the X-ray emission originates from a collection of point sources, by far the most likely interpretation is that of a poor galaxy cluster. This is supported by the finding of four coincident galaxy redshift in the optical follow-up.

RXCJ0117.8-5455 is another source with X-ray emission consistent with a point source origin. The source is also detected as a bright radio source in the SUMSS survey (Mauch et al. 2003). Since the cluster is found to be at a redshift of $z=0.2510$ as determined from 6 galaxy velocities (where none of the spectra indicate an AGN), an upper limit on the X-ray core radius of about $270 h_{50}^{-1} \mathrm{kpc}$ is consistent with a cluster source and also the spectral hardness ratio is within the limits expected for cluster emission. Therefore this point-like X-ray source is kept in the catalogue until we have better data for a more secure identification.

RXCJ0132.6-0804 is classified as Seyfert 1.5 in the cross correlation work of the ROSAT All-Sky Survey and the NVSS sources by Bauer et al. (2000). We find narrow emission lines in the central galaxy, PKS 0130-083, of this cluster at $z=0.1485$. The [OIII] lines are more prominent than the $\mathrm{H} \beta$ lines which makes the AGN identification more likely than that of the typical emission lines often found in central cluster galaxies in cooling core clusters (e.g., Crawfrord et al. 1999). The X-ray luminosity of the object is about $3.6 \times 10^{44} \mathrm{erg} \mathrm{s}^{-1}$ which is quite high for a Seyfert galaxy. The Digital Sky Survey image shows clearly a central dominant galaxy surrounded by a collection of galaxies and we recorded three coincident galaxy redshifts. The spectral hardness ratio is within the limits expected for thermal emission from a cluster. Therefore the identification of this X-ray source is uncertain at this stage. The $\mathrm{X}$-ray emission could come from the cluster or the AGN. A high resolution X-ray observation is required for a definitive decision.

RXCJ0250.2-2129 has been classified as a BL Lac $(z=$ 0.4980 , off-set 0.4 arcmin) in the ROSAT Bright Source Catalogue by Schwope et al. (2000) and is listed as a radio source in Bauer et al. (2000). The BL Lac is not at the same 


\section{H. Böhringer et al.: The REFLEX cluster catalogue, Online Material p 3}

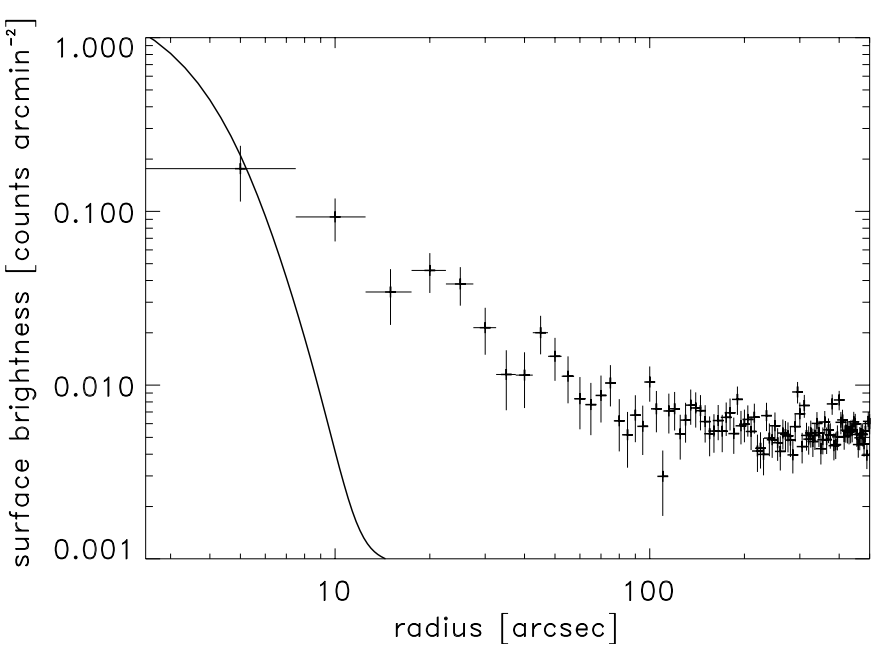

Fig. A.1. Surface brightness profile of the REFLEX cluster RXCJ0301.6+0155 as observed in a pointed observation with the ROSAT HRI (data points and Poissonian errors). The profile is compared to the point spread function of the ROSAT HRI (solid line).

redshift as the cluster. The X-ray source shows a marginal extent and is too soft by about $1.3 \sigma$ compared to the expectation. Our deeper CCD image clearly shows an optical cluster and we found two coincident galaxy redshifts. We therefore take the cluster identification as more likely, but cannot rule out a contribution to the X-ray emission by the AGN. The cluster is also coverd by a ROSAT PSPC archive observation. But the offset from the pointing center is so large that the possibility to better resolve a point source is not improved over the survey data.

RXCJ0301.6+0155: This source, which has previously been identified as a cluster in the NORAS Survey (Böhringer et al. 2000) and which is coinciding with a Zwicky cluster (ZwCl0258.9+01), appears point-like. A ROSAT HRI observation in the archive showed that the source is very compact but definitely not a point source. Therefore the cluster identification is well justified. The comparison between the surface brightness profile and the point spread function of the ROSAT HRI is shown in Fig. A.1.

RXCJ0331.1-2100 is coincident within 0.5 arcmin with a Seyfert 1.9 galaxy identified by Bauer et al. (2000). The source is listed in Schwope et al. (2000) as RBS 0436. The $\mathrm{X}$-ray emission is only marginally extended, but the spectral hardness ratio is consistent with thermal intracluster medium emission. We have nine coincident galaxy redshifts for this cluster. Therefore it is possible that the AGN is contaminating the X-ray radiation, but there is also definitely a cluster at this position.

RXCJ0336.3-0347: This is a compact X-ray source with a peaked center and a weak extent. The central galaxy is listed as an AGN, as 2MASS and radio source, probably a BL Lac at $z=0.1595$ (Bauer et al. 2000; Veron-Cetty \& Veron 2001). It appears to have a bright central spot in sky images, but our spectra also easily show the underlying galaxy continuum with the Balmer break. The optical image also shows an optical cluster for which we have three concordant redshifts at the same redshift as the AGN. The most likely interpretation of this source is that it is indeed a galaxy cluster with some (less than half) contamination of the X-ray emission by the AGN. The hardness ratio of the X-ray emission is consistent with a cluster, also.

RXCJ0425.8-0833 is coincident within 1.3 arcmin with a Seyfert 2 galaxy identified by Hewitt \& Burbidge (1991). The source is listed in Schwope et al. (2000) as RBS 0540. The central dominant galaxy has the spectrum of a passive elliptical galaxy without emission lines as found in our followup observations. The X-ray emission is very extended and the dominant part of the X-ray emission must come from the cluster, for which we have two concordant redshifts.

RXCJ0437.1-2027, A499, is detected with only 26 photons and the result for the extent is not completely convincing. A ROSAT HRI observation from the archive shows that the source is quite compact but clearly extended. Two coincident galaxy redshifts are available for this cluster.

RXCJ0528.9-3927 also identified as RBS0653 (Schwope et al. 2000) is listed in NED as a QSO. The X-ray emission is slightly extended but the spectral hardness ratio deviates by $5.2 \sigma$ from the value expected for thermal cluster emission. A deeper X-ray observation with XMM-Newton shows this object to be an X-ray luminous cluster with a less than $20 \%$ contamination (in the ROSAT hard band) by a bright, soft AGN point source.

RXCJ0918.1-1205, A780 or Hydra A, is a well known $\mathrm{X}$-ray cluster. Thus even though one finds a coincidence within 0.2 arcmin with a Seyfert galaxy listed by de Vaucouleurs et al. (1991), there is no doubt about the clear dominance of the thermal cluster emission.

RXCJ1027.9-0647, A1023, is coincident within 0.2 arcmin with an AGN at $z=0.1165$ identified by Grazian et al. (2002). The X-ray emission is marginally extended and the spectral hardness ratio is consistent with thermal cluster emission. There is a residual possibility that the X-ray emission of this source is contaminated by an AGN. Six coincident galaxy redshifts support the existence of a galaxy cluster at this position.

RXCJ1050.5-0236, A1111, is coincident within 0.6 arcmin with a Seyfert 2 galaxy identified by Machalski \& Condon (1999). The X-ray emission is found to be extended and the dominant part of the X-ray emission comes from the cluster. There is an X-ray emitting star in the south of the cluster whose X-ray emission was deblended. Nine coincident galaxy redshifts leave no doubt about the existence of a cluster at this position (see also the ASCA study of Matsumoto et al. 2001).

RXCJ1050.6-2405 features a point source and an extended fainter halo in the RASS. About one third of the flux seems to come from the central compact emission. The hardness ratio gives no indication for two source components, however, and is perfectly consistent with thermal emission from a cluster. At the cluster redshift of $z=0.2037$ the compact center could still be a bright compact cluster cooling core as well as a contaminating central AGN. Near the center ( 0.4 arcmin offset) is a radio source, PKS B1048-238 (MRC1048-238), at a redshift of $z=0.2060$ (McCarthy et al. 1996) and identified with a broad line radio galaxy Kapahi et al. (1998). We expect that most of 
the X-ray emission is due to the cluster. But so far we have made no effort to subtract the possible AGN contribution.

RXCJ1141.4-1216, A1348, is coincident within 0.2 arcmin with a Seyfert 1.8 galaxy listed by Machalski \& Condon (1999). The X-ray emission shows a small extent and the X-ray emission is slightly softer $(\sim 2.9 \sigma)$ than expected. From the extended X-ray emission we still conclude that most of the emission comes from the cluster. The X-ray luminosity of the object is $3.3 \times 10^{44} \mathrm{erg} \mathrm{s}^{-1}$ which would be towards the upper end of the luminosity distribution of Seyfert galaxies. Six coincident galaxy redshifts confirm the existence of a cluster at this position.

RXCJ1149.7-1219, A1391, is coincident within 0.9 arcmin with an AGN listed by Machalski \& Condon (1999). The $\mathrm{X}$-ray emission is found to be clearly extended and the dominant part of the X-ray emission must come from the cluster. Six coincident galaxy redshifts confirm the existence of a cluster at this position.

RXCJ1212.3-1816 was detected with only 12.5 source photons with a significance of just about $3 \sigma$. This signal is detected in an aperture significantly larger than the point spread function and the significance decreases for a smaller detection cell. Therefore, if the detection is accepted, the source has to be extended. We have two coincident cluster galaxy redshifts.

RXCJ1234.2-3856 appears as a point source in RASS with a spectral hardness ratio consistent with thermal cluster emission. It is also listed as a radio source without classification by Bauer et al. (2000). Two coincident galaxy redshifts give some further support for the cluster identification.

RXCJ1253.6-3931 appears as point-like source in the RASS with a cluster like hardness ratio and coincides within 0.5 arcmin with the unclassified radio source PMN J1253-3932 (see also Bauer et al. 2000). We have a spectrum as well as a redshift from the central galaxy which does not show an AGN or BL Lac signature. A CCD image shows a galaxy cluster. Therefore we keep this source in our list as a likely cluster candidate.

RXCJ1326.2+0013 is coincident within 0.2 arcmin with a BL Lac found in the $2 \mathrm{dF}$ survey. The X-ray emission is clearly extended and the emission from the intracluster medium certainly dominates. The spectral hardness ratio is as expected and there are 16 coincident galaxy redshift. Therefore there is no doubt about the cluster identification of this source.

RXCJ1332.9-2519 is detected in the RASS with very low surface brightness, but with 50 counts and an about $4 \sigma$ detection. An archival PSPC observation, with an exposure of only $982 \mathrm{~s}$ shows a similar very low surface brightness structure providing a good confirmation of this very diffuse structure. We have three coincident galaxy redshifts from the follow-up observations confirming the existence of a cluster at this position.

RXCJ1347.2-3025 and RXCJ1349.3-3018, A 3574 or Klemola 27 at a redshifts of 0.0145 and 0.0160 , respectively, is a cluster with two components. It presents a particular problem to its detection and identification. The central galaxy is the X-ray luminous Seyfert 1 galaxy, IC 4329, and two further AGNs contribute to the X-ray emission from the cluster region. While the central detection could easily have been dismissed because of its almost point-like appearance in the

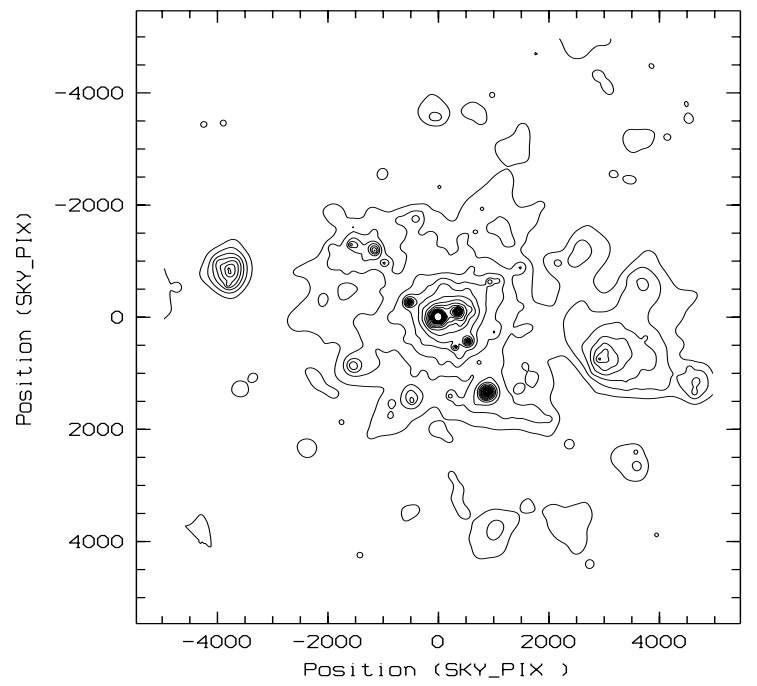

Fig. A.2. ROSAT PSPC image of Abell 3574 (RXCJ1347.2-3025 and RXCJ1349.3-3018) from a pointed PSPC observation with an exposure of $8.3 \mathrm{ks}$. The image is exposure and vignetting corrected. It has been treated with a variable Gaussian filter. The contours are logarithmically spaced. We can see two bright point sources in the center and another one to the south which carry most of the flux received from this object. The diffuse emission of the cluster shows some clumpiness and amounts to about $20 \%$ of the total emission. The central part and the Western subcluster are tabulated as two different catalogue entries. One sky pixel is $0.5 \mathrm{arcsec}$, thus the image size is $42 \times 42 \mathrm{arcmin}^{2}$. The image is centered on the sky coordinate 1349 19.2, -30 1836 (J2000).

RASS and its identification with a known X-ray AGN, the additional detection of fragments of the diffuse outer emission in the RASS analysis triggered a further inspection of this cluster. A pointed observation shows clearly the diffuse $\mathrm{X}$-ray halo of the cluster in addition to the three cluster AGN point sources. We have used this pointed observation to subtract the contribution of the three AGN from the total emission. The central AGN IC4329A carries about $75 \%$ of the flux, the two other AGNs contribute about 4 to $5 \%$ each and the cluster emission amounts to $15-20 \%$. Therefore, with a net flux of $\sim 1.35 \times 10^{-11} \mathrm{erg} \mathrm{s}^{-1} \mathrm{~cm}^{-2}$ in the ROSAT band, the cluster is well above the flux limit of the REFLEX and the XBACS survey. Figure A. 2 shows the surface brightness distribution of this cluster. In the catalogue we have listed the central cluster region and the western concentration (about 25 arcmin, $\sim 0.6 \mathrm{Mpc}$ distance) as separate sources identified with A3574E and $\mathrm{A} 3574 \mathrm{~W}$, respectively, recognizing that they are two separate virializing clumps. Also the diffuse Western component is, with a nominal flux, $F_{\mathrm{n}} \sim 3.5 \times 10^{-12} \mathrm{erg} \mathrm{s}^{-1} \mathrm{~cm}^{-2}$, above the REFLEX flux limit.

The cluster properties were determined also with the help of the pointed observation, which allows a better contamination subtraction. The cluster was dismissed in XBACS as an AGN. At a larger distance it would probably have escaped its identification as a cluster in REFLEX. This shows that there is probably not only a small fraction of clusters which are erroneously included in the sample because of an AGN contribution to the $\mathrm{X}$-ray flux, but also a small fraction of clusters which are er- 
roneously discarded because the cluster X-ray emission is not easily visible if it is blended by a bright AGN.

RXCJ1415.2-0030, tentatively identified with A1882 (offset $13.3 \mathrm{arcmin}$ ), is coincident within 1.6 arcmin with a QSO identified in the Sloan Digital Sky Survey. The X-ray emission is very extended and diffuse, however, and we do not detect a contribution by a point source. 20 concordant redshifts confirm the existence of a cluster.

RXCJ1416.8-1158 appears as a point source in RASS and has a spectrum that is $1.7 \sigma$ too soft. 10 coincident galaxy redshift give good support for the existence of a cluster at this position.

RXCJ1504.1-0248 is a very peculiar object. We have obtained a spectrum of the central galaxy (with an offset of 0.3 arcmin from our reference position of the cluster) which shows Liner like emission lines. It was identified as an AGN by Shectman et al. (1996). The X-ray source is very compact but significantly extented (the probability that the emission comes from a point source is estimated to be 0.0016). The X-ray luminosity is extremely high with $L_{\mathrm{x}} \sim 4.3 \times 10^{45} \mathrm{erg} \mathrm{s}^{-1}$ in the $0.1-2.4 \mathrm{keV}$ band and not typical for a narrow line AGN.

A short CHANDRA observation that was finally performed early 2004 shows a perfect galaxy cluster image without any significant AGN contamination. This is in our sample the most spectacular example of an object which was for long time marked as very suspicious for its unusual compactness for given brightness and distance but was finally revealed to be a prominent X-ray cluster. The Liner-like spectrum observed for the central galaxy is also typical for cooling flow clusters (e.g., Crawford et al. 1999, and references therein).

RXCJ1958.2-3011 is an X-ray source that appears pointlike in RASS with a hardness ratio consistent with cluster emission. It is listed as an unclassified radio source in Bauer et al. (2000). The optical image shows a galaxy with a bright nucleus and our ESO spectrum indicates an enhanced blue continuum. There is clearly a cluster visible with a likely Bright Cluster Galaxy (BCG) that is about 1 arcmin offset to the NEE from the radio source and the center of the cluster emission. The cluster detection is further supported by four concordant redshifts. Therefore, we classify this object as a cluster-AGN combination whose fluxes must be determined by a higher angular resolution X-ray image. Since low X-ray luminosity AGN appear very frequently in clusters, with an X-ray luminosity well below that of the cluster emission, we keep this object in the REFLEX catalogue at present.

RXCJ2035.7-2513 shows diffuse X-ray emission around a large early type galaxy with a luminosity of about $4 \times$ $10^{42} \mathrm{erg} \mathrm{s}^{-1}$. An archival PSPC image also shows the diffuse emission but also some contamination of $10-20 \%$ by point sources, which is within the error of the flux determination in the catalogue and no correction has been made. The object is probably associated with A3698 whose center has an offset of 4.6 arcmin assumed to coincide with NGC 6936. Seven coincident galaxy redshifts confirm the existence of a cluster at the $\mathrm{X}$-ray source position.

RXCJ2147.0-1019 contains a BL Lac identified by Bauer et al. (2000) within 0.2 arcmin of our reference position. The $\mathrm{X}$-ray source is marginally extended (probability 97\%) and has a hardness ratio well consistent with thermal cluster emission. We cannot rule out that the BL Lac could substantially contribute to the cluster emission, however.

RXCJ2216.9-1725 contains a Seyfert galaxy identified by Schwope et al. (2000) within 0.2 arcmin of our reference position. The X-ray source is marginally extended (probability $90 \%$ ) and the X-ray hardness ration shows that the source is about $2 \sigma$ too soft. Therefore, it is not impossible that the Seyfert galaxy contributes to the $\mathrm{X}$-ray emission. The cluster is with an X-ray luminosity of $L_{\mathrm{x}} \sim 4 \times 10^{44} \mathrm{erg} \mathrm{s}^{-1}$ so luminous that the Seyfert galaxy has to be relatively bright to affect the total flux.

RXCJ2220.5-3509, A3866, has an AGN found in our spectroscopic follow-up 40 arcsec from the X-ray center at $z=0.0754$. The $\mathrm{X}$-ray emission shows a small but significant extent, but the core of the emission looks like a point source. The cluster redshift is measured as $z=0.1544$ and the optical image shows a nice cluster with a dominant giant galaxy close to the X-ray maximum. We expect that the X-ray emission from the cluster is partly contaminated by the AGN.

RXCJ2234.5-3744, A3888, is a cluster in which we also detected a Seyfert 1 galaxy within 2 arcmin from the center (Romer 1994, Ph.D. Thesis). Therefore, there was some concern about the X-ray contamination from the AGN in this source. An XMM-Newton observation (P.I. A.C. Edge) allows us to distinguish the cluster and AGN X-ray emission. We find that the AGN contribution is about $10 \%$ of the total emission in the $0.5-2.0 \mathrm{keV}$ band. 70 coincident galaxy redshifts confirm the existence of a cluster at this position.

RXCJ2251.7-3206 is an X-ray source that appears pointlike in RASS with a hardness ratio which is too soft by about $2.9 \sigma$ compared to the expectation for cluster emission. It has been found to be a radio source (Bauer et al. 2000) classified as a BL Lac (Schwope et al. 2000). We have one galaxy redshift for this cluster. The question if it is an X-ray BL Lac or an X-ray cluster is undecided. This is to be clarified by further observations.

RXCJ2351.6-2605 contains an AGN within 0.2 arcmin of our reference position identified by Caccianiga et al. (2000). The X-ray source is, however, extended with high significance, and the X-ray hardness ratio is perfectly consistent with thermal emission from a cluster. We do not detect a signature of point source contribution. Therefore, the identification of this $\mathrm{X}$-ray source as a cluster is safe inspite of the coincidence with the AGN.

For some of the objects in this list, where the central cluster galaxy is identified with an AGN because of the observation of emission lines, the emission lines could also be associated to the cluster cooling core. Emission lines with preferentially low excitation energies are frequently observed in cooling core clusters (e.g., Crawford et al. 1999, and references therein). In these cases the dominant X-ray emission comes from the cluster and its cooling core. Therefore the observation of emission lines in the central cluster galaxies does not cast doubts on the cluster identification in general. 


\section{A.1. Objects removed from the present catalogue at final inspection}

In the final two observing runs a number of cluster candidates were observed which had been flagged to be weak cluster candidates. A large fraction of them turned out to be clusters at the telescope and were therefore included in the catalogue. Now, during this final inspection a few of them turn out to be most probably optical clusters with a dominant X-ray AGN. In the following we list these 7 objects which were excluded from our catalogue.

RXCJ0730.8-6602 looks like a point source. The central galaxy with a redshift of $z=0.1063$ could be an AGN; it has a bright core in the optical image. While the DSS image shows a trace of a promising galaxy grouping at the center of the X-ray position, a deeper $R$-band CCD did not confirm the presence of a rich enough cluster. The Parks $4.85 \mathrm{GHz}$ survey lists a $84 \mathrm{mJy}$ radio source, PMN0730-6602, (Griffith \& Wright 1993) and the SUMSS survey (Mauch et al. 2003) as a $81.7 \mathrm{mJy}$ source at $485 \mathrm{MHz}$. Therefore we have removed this object from the final catalogue and classify it tentatively as an X-ray AGN, possibly a BL Lac, within a galaxy group, where the X-ray emission comes preferentially from the AGN.

RXCJ0934.4-1721 is a source with a marginal X-ray extent, but a hardness ratio consistent with thermal cluster emission within $1 \sigma$. There is no striking galaxy overdensity in deeper optical images and no clear central BCG. A galaxy close to the center with a redshift of $z=0.2499$ was identified as a BL Lac candidate by Bauer et al. (2000) in the ROSAT-NVSS correlation sample. Therefore, this object is most probably not a cluster and was removed from our cluster catalogue.

RXCJ1046.8-2535 looks like a point source in the RASS and is confirmed to be a point source in a ROSAT HRI observation. Therefore, a cluster identification is ruled out for the dominant fraction of the X-ray emission. Nevertheless we find an optical cluster at redshift $z=0.2426$ with 8 coincident galaxy velocities. The source is also listed as a radio source in Condon et al. (1998) and Bauer et al. (2000). There is an indication in the HRI observation that there is a faint halo underneath the point source with a flux of at most $5 \%$ of that of the central source, which could be the emission from the cluster. The flux is, however, more than an order of magnitude below the REFLEX limit and therefore the cluster was removed from the REFLEX sample. This object falls most probably into the category of X-ray AGN in a galaxy group or cluster.

RXCJ1213.3-2617 is coincident within 0.5 arcmin with a BL Lac identified by Fischer et al. (1998). Our spetrum of the central galaxy does not show an AGN or BL Lac signature, however, and a CCD image provides some indication of a cluster. The X-ray emission is not significantly extended and the spectral hardness ratio is consistent with thermal cluster emission. A short ROSAT HRI exposure $(2.7 \mathrm{ks})$ shows only a point source, whose flux corresponds only to about 0.13 PSPC counts $\mathrm{s}^{-1}$ compared to 0.244 observed in the survey. There is no signature of further extended emission in the HRI image. Therefore the most likely interpretation of this X-ray source is an AGN which has shown a dimming by a factor of 1.8 between the two ROSAT observations.

RXCJ1545.7-2339 appears as point-like in RASS, but has a reasonable hardness ratio for cluster emission. The archival HRI data show that more than $90 \%$ of the flux comes from a point source. Since the total flux of the source is only about twice above the REFLEX flux limit and since the cluster emission for this object at a redshift of $z=0.1205$ should well be extended at the HRI resolution, we remove this source from the REFLEX sample. Bauer et al. (2000) list this as a radio source without further classification, making an AGN counterpart likely, but our ESO spectroscopic observations provided no evidence for an AGN. Two coincident galaxy redshifts found make it likely that the AGN resides in a group or cluster.

RXCJ2040.0-7114, tentatively identified with the cluster A3701 at redshift $z=0.1607$, is an X-ray source that appears point-like in the RASS with a hardness ratio consistent with cluster emission. An archival ROSAT HRI observation shows a surface brightness distribution with a bright point source and a faint, small halo with an upper limit on the flux contribution of only a few percent. This brings this X-ray source well below the REFLEX flux limit and we removed this source from the REFLEX catalogue. An $R$-band CCD image shows a nice galaxy cluster with two bright central galaxies, which is no surprise as it was already classified as a cluster by Abell et al. (1989). Our spectroscopic follow-up provides 12 coincident galaxy redshifts confirming the cluster detection. Therefore, this object falls most probably into the category of $\mathrm{X}$-ray luminous AGN in a galaxy cluster.

RXCJ2041.8-3733, tentatively identified with the cluster S892 at redshift $z=0.0997$ (offset $\sim 5 \operatorname{arcmin}$ ), is an X-ray source that appears point-like in the RASS with a hardness ratio which is too soft by about $1.8 \sigma$ compared to the expectation for cluster emission. An archival ROSAT HRI observation shows only a point source. Our spectroscopic follow-up observations show a Seyfert 1 spectrum for the central galaxy and provide 8 further coincident galaxy refdshifts. A deep $R$-band image shows a cluster with an appearance consistent with the determined redshift around $z=0.1$ and the central galaxy appears to have a bright core. At the measured redshift this cluster should appear clearly as an extended X-ray source in the RASS and definitely in the HRI image. Therefore we identify the main X-ray emission with the AGN and removed this object from the REFLEX catalogue.

The source is also listed in the bright RASS-NVSS correlation by Bauer et al. (2000) as an unclassified radio source and as a cluster in the ROSAT Bright Survey (Schwope et al. 2000). 